\title{
PERSPECTIVA PALEOBOTÁNICA Y GEOLÓGICA DE LA BIODIVERSIDAD EN MÉXICO
}

\author{
Sergio R. S. Cevallos-Ferriz ${ }^{1,3}$, Enrique A. GonzÁlez-Torres ${ }^{1,2}$ \\ y Laura CALvillo-CANadell ${ }^{1}$
}

\begin{abstract}
${ }^{1}$ Universidad Nacional Autónoma de México, Instituto de Geología, Departamento de Paleontología, Ciudad Universitaria, 04510 Coyoacán, México D.F., México.

${ }^{2}$ Dirección actual: Universidad Nacional Autónoma de México, Facultad de Ingeniería Geológica, Coordinación de la Carrera de Ingeniería Geológica, Ciudad Universitaria, 04510 Coyoacán, México D.F., México.

${ }^{3}$ Autor para la correspondencia: scrscfpb@unam.mx
\end{abstract}

\section{RESUMEN}

El origen de la vegetación actual de México y su diversidad tiene larga historia. Posiblemente es la extensión de esta historia el punto en que discrepan las propuestas, una planteando que inicia en el Cretácico (ca. $132 \mathrm{ma}$ ) y otras haciendo énfasis en procesos restringidos al Plio-Pleistoceno (5.3 ma), sobre todo si se refieren al origen de la vegetación actual. El aumento del conocimiento sobre la evolución geológica de México, y del constante cambio en su fisiografía, así como del estudio de las angiospermas fósiles de la región, genera un concepto más claro de cómo y cuándo las formas de vida fueron llegando y asociándose. Se presenta una hipótesis en la que se combinan procesos geológicos y cambios fisiográficos, con la presencia de plantas y vegetación en las partes emergidas que se van desarrollando. Se propone que la biodiversidad actual efectivamente inicia hace ca. 132 millones de años, aunque linajes que hoy viven en México se pueden reconocer desde este tiempo, es complicado ubicarlos en familias, pues posiblemente representen miembros del grupo troncal. En el Paleógeno (65-32 ma) las familias, y aun géneros, que continúan viviendo en el país son más fácilmente reconocidos, pero grupos extintos o que hoy crecen en otras regiones siguen siendo comunes. Es en el Neógeno (32-1.8 ma) que desde un punto de vista de la morfológico/anatómico las plantas fósiles se parecen más a las que viven de forma natural actualmente en el país, pero muestran diferencias que en general permiten proponer nuevas especies. Si las plantas fósiles y actuales de México se relacionan morfo/ anatómicamente más solo en tiempos relativamente recientes, es de esperar que con los tipos de vegetación suceda algo similar. El registro fósil sugiere que a partir de comunidades 
que se desarrollaron bajo condiciones cálido-húmedas en el Cretácico, divergieron tipos de vegetación con capacidades diferentes ante el estrés hídrico, y comunidades que se favorecieron de condiciones templadas a frías. Esto sucede aparentemente en dos momentos distintos en dos regiones diferentes; durante el Paleógeno se afecta al norte y en al Neógeno al centro y sur del país. Trabajo geológico y paleobotánico conjunto y comparativo permitirá refinar esta propuesta que sugiere que los cambios que activan o restringen respuestas biológicas forman parte de otros componentes del Sistema Tierra.

Palabras clave: geología, historia de la vegetación, México, Paleobotánica, procesos geobiológicos.

\section{ABSTRACT}

The origin of the extant vegetation and diversity of Mexico has a long history. Possibly the extent of this history is the point where the proposals differ, one states that the history begins in the Cretaceous (ca. 132 my) while other emphasize processes restricted to the Plio-Pleistocene (5.3 my), especially if referring the appearance of extant vegetation. Increased knowledge of the geological evolution of Mexico, and the constant change in physiography, along with the study of fossil angiosperms of the region, generates a clearer concept of how and when life forms differentiated and partnered. A hypothesis that combines geological processes and physiographic changes with the presence of plants and vegetation on the emerged continental parts is presented. It is proposed that existing biodiversity does indeed start ca. 132 million years ago, and although lineages that live in Mexico today can be recognized from this time, it is difficult to place their representants within a family, probably because they may represent members of the stem group. By the Paleogene (65-32 my) families, and even genera, that continue to live in Mexico are more easily recognized, but groups of extinct plants or plants growing today in other regions are still common. Based on the morphological/anatomical approach the Neogene (32-1.8 my) fossil plants resemble more those living naturally in Mexico, but still have important differences supporting the recognition of new species only. If in relatively recent times extant and fossil plants in Mexico seem closer based on their morphology/anatomy, it is to be expected that with the vegetation types a similar process can be recognized. The fossil record suggests that from communities that developed under hot-humid conditions in the Cretaceous, vegetation types with different capabilities to tolerate water stress, and communities that favored cool temperate conditions diverged. The process of divergence apparently occurred at two different times in two different regions. During the Paleogene it affected northern Mexico and during the Neogene 
it affected central and southern Mexico. Further geological and paleobotanical work will refine this proposal that suggests that changes that enable or constrain biological responses are part of other components of the Earth System.

Key words: geobiological processes, geology, Mexico, Paleobotany, vegetation history.

\section{INTRODUCCIÓN}

Los cambios biológicos que se han dado a través del tiempo, se pueden comprobar de manera diversa; por ejemplo por medio de los múltiples estudios numéricos que hoy son ampliamente utilizados y que han incentivado el entendimiento de muchos aspectos de la vida y sus procesos. Sin embargo, entre las observaciones históricas siguen siendo aquellas de las Ciencias de la Tierra, incluyendo a la paleontología y paleobiología, las únicas fuentes directas a través de la cual se pueden comprobar las hipótesis que se generan a través de estas investigaciones (e.g., Nixon, 1996; Bateman y DiMichele, 2003; Bradley et al., 2003, Crane et al., 2004; Lewis, 2006; Hilton y Bateman, 2006 ). Lo anterior lo podemos ejemplificar con datos que requirieron de muchos años de observación y experimentación. Las hipótesis sobre cómo eran los primeros organismos solo lograron comprobarse cuando se encontraron microfósiles en rocas del Precámbrico, y en la actualidad se continúan examinando meteoritos con el fin de poder encontrar también evidencia de vida extraterrestre (e.g., Russell, 2006; Schopf, 2006; Burns et al., 2009). Ha sorprendido la radiación de organismos que tuvo lugar hace ca. 570 millones de años en la que se encuentran muchos phyla, entre los cuales algunos son taxa nuevos y otros aunque sí se insertan en grupos conocidos, han sido difíciles de ubicar taxonómicamente (e.g., Dornbos y Xiao, 2008; Maruyama y Santosh, 2008). Otro ejemplo se encuentra al estudiar a las primeras plantas que colonizaron las partes emergidas de los continentes, mismas que aún actualmente son objeto de discusiones acaloradas en las que se exponen argumentos que se contraponen. No hace mucho se explicaba que las primeras plantas tenían que haber desarrollado raíz, hojas, tejido de conducción y epidermis; pero en las últimas décadas se han encontrado fósiles que muestran que no tenían raíces sino rizomas, carecían de hojas, iniciaron con enaciones, su tejido vascular tenía elementos conductores sin pared secundaria y solo después fueron adquiriendo la lignina. Así, el concepto actual de planta vascular se complementa de forma importante con la observación directa de los fósiles (e.g., Bonfante y Selosse, 2010; Weng y Chapple, 2010). 
Ya en el Carbonífero (hace ca. $355 \mathrm{ma}$ ), ejemplos de cambios en la morfología y anatomía de las plantas sugieren variaciones en los procesos fiiológicos y en las interacciones entre organismos, algunas distintas a las de los organismos actuales. En ese tiempo todavía existían plantas con embriones unipolares, es decir no contaban con meristemo subapical y no producían raíces (e.g., Lepidondendrales, Calamitales), y muchas de estas no crecían en diámetro como lo hacen las plantas actuales, tenían un cambium vascular unifacial, aparentemente no producían floema. El xilema secundario era escaso comparado con el volumen de corteza que frecuentemente contenía cordones de fibras para reforzar a este tejido que sostenía a la planta (e.g., Lepidondendrales, Calamitales) (e.g., Phillips y DiMichele, 1992; Linkies et al., 2010). Para entonces las semillas ya eran un componente novedoso de otros grupos de plantas, pero solo el registro fósil pudo mostrar que antes de la adquisición de la reproducción gimnospérmica con semillas desnudas, una serie de plantas extintas (e.g., Pteridospermas) exhibieron un tipo de reproducción anemófilo en el que los granos de polen eran capturados gracias a remolinos generados por el movimiento del salpnix/lagenostoma, modificaciones de la nucela que se proyectaban más allá del micrópilo, dirigiendo a los granos de polen a la cámara polínica (e.g., Long, 1977; Stubblefield y Rothwell 1980; Stubblefield et al., 1984; Sanders et al., 2009).

Un último ejemplo referente a los cambios que inciden en la forma en que la vida se relaciona con ella misma y su entorno se encuentra al estudiar el origen de las plantas con flor. Antes de ellas, el color sobre el continente dado por las plantas era probablemente poco variado, dominaban los tonos verdes y pardos reflejados por las hojas y tallos, aunque por ahí pudo haber estructuras con algún otro matiz, estas no eran abundantes. Entonces variados pigmentos se hicieron aparentes hace ca. de 132 millones años revolucionando las interacciones, originando diversas adaptaciones en los insectos, a los animales les cambió el paisaje, la vista pudo discriminar con ayuda del espectro de luz, y la coloración generó microhábitats. Además, las plantas con flor hicieron más importante la retribución a los animales que les visitaban otorgando azúcares y otros alimentos a quienes les asistían en la polinización y dispersión. Estas plantas también perfumaron al mundo, los olores se convirtieron en mensajeros y atrayentes (e.g., Feild y Arens, 2007; Frohlich y Chase, 2007; von Balthazar et al., 2008; Pennisi, 2009; Smith et al., 2010).

Así, los cambios en la vida desencadenan variantes para aprovechar recursos, promover su uso, y/o generar interacciones. Los pastizales y manglares actuales dominados por angiospermas, pueden complementar esta forma de entender los cambios. ¿Es válido preguntarse si antes de la aparición de los biomas actuales existían otros biomas? ¿Serían los helechos los encargados de formar grandes praderas? o 
¿las gimnospermas tendrían plantas que ocupaban el nicho de los manglares? Son los fósiles quienes aportan evidencias para responder a estas preguntas. Por ejemplo, se ha documentado que a falta de pastos existieron grandes extensiones de terrenos dominadas por helechos en el Mesozoico, y que algunas Cheirolepidaceae (coníferas extintas) formaban comunidades semejantes a los mangles actuales (Collinson, 2002; Van Konijnenburg-Van Cittert, 2002; Taylor et al., 2009; Wilson, 2009), pero en cada momento las relaciones entre los organismos eran distintas, esto seguramente debido a que son diferentes los participantes aunque las situaciones son semejantes.

Las preguntas y respuestas pudieran seguir, pero hay que documentar las aportaciones del registro fósil al entendimiento del origen de la biodiversidad actual en México. Aunque el conocimiento y entendimiento de las plantas que vivieron en el pasado en nuestro territorio es ahora más completo y está mejor fundamentado, el conocimiento de la geología de las regiones continentales expuestas para México es más restringido ya que solo existen pocos esfuerzos, en el mejor de los casos superficiales, por exponer cómo estos interactuaron para permitir que se estableciera la mega diversidad que hoy caracteriza al país (e.g., Maldonado-Koerdell, 1950; Weber y Cevallos-Ferriz, 1995). Añadir la información paleobotánica a las hipótesis sobre generación de la biodiversidad enriquecerá la propuesta y ampliará la forma de abordar el problema. Para ello hay que separar dos visiones de la historia de la biodiversidad. Primero aquella que tiene que ver con la evolución de los linajes y que puede en este caso iniciarse en el Cretácico; y otra, relacionada con el origen de la vegetación actual que indudablemente tiene que ver con la "modernización" de los taxa que forman parte de la primera, en donde la perspectiva histórica es mayor.

Se ha ejemplificado cómo los organismos cambian y a su vez cómo estos últimos repercuten en la forma de funcionar e interactuar, además de que se distribuyen de acuerdo con las características del medio físico, mismo que es moldeado y modificado continuamente por la actividad geológica. Es también ampliamente aceptado que la Tierra ha sufrido una diversidad de cambios a lo largo de su historia (e.g., Lieberman, 2000). Cualquier punto que observemos en la Tierra tiene una historia larga que en términos sencillos se puede resumir diciendo que ha cambiado de latitud, longitud, y/o altitud, y esto conlleva cambios en temperatura, precipitación, presión, etc. Esta actividad implica procesos importantes, como por ejemplo los involucrados en la formación de cadenas montañosas. Seguramente los cambios y sus efectos serán distintos en una región con gran actividad tectónica como el este de México, en comparación con otras caracterizadas por una significativa actividad volcánica como el oeste y centro, y aun sur del país (Morán-Zenteno, 1984). Los suelos que se formen en uno y otro lado tendrán patrones de evolución distintos; probablemente 
en los sitios con gran vulcanismo la cubierta edáfica no completará sus ciclos de vida y continuamente se reiniciarán, mientras que en otros lugares estos serán más completos y los organismos que establezcan relaciones con los suelos serán seleccionados por esta actividad (Jasso-Castañeda et al., 2002, Royer, 2010). Entendiendo a estos procesos y sus consecuencias como modificadores de los escenarios, se puede reconocer su influencia directa en los seres vivos, disparando la acción de procesos biológicos generadores de diversidad. Concibiendo de esta forma a los escenarios geológicos, geomorfológicos edáficos, etc., en las siguientes páginas comentaremos algunos aspectos de las plantas que fueron estableciéndose en ellos.

\section{MATERIAL Y MÉTODO}

Este trabajo se basa en la recopilación de datos e ideas presentadas por colegas interesados en el entendimiento de lo que llamamos actores (organismos) y escenarios (fisiografía) modelados por los procesos geológicos, así como en nuestra experiencia al querer explicar la presencia de plantas en momentos distintos en la historia de la vegetación de México. La parte geológica es particularmente interesante por la información generada por colegas ocupados en documentar la formación de las calderas de la Faja Volcánica Transmexicana, en donde la interacción de lo geológico y biológico es relevante. Con el fin de conocer a los fósiles que se mencionan a lo largo de este trabajo se recomienda consultar las fuentes originales (e.g., Cevallos-Ferriz, 1983; Cevallos-Ferriz y Weber, 1992; Rodríguez de la Rosa y Cevallos-Ferriz, 1994, Rodríguez de la Rosa y Cevallos-Ferriz, et al., 1998; Hernández-Castillo y CevallosFerriz, 1999; Calvillo-Canadell y Cevallos-Ferriz, 2005, 2007; Estrada-Ruiz et al., 2010; Calvillo-Canadell et al., sometido). Para ampliar la visión sobre la paleovegetación de México y conocer a través de fotografías plantas del pasado, evitamos ilustrar aquí fósiles previamente descritos y usamos ejemplares que actualmente están siendo estudiados por el grupo de Paleobotánica del Instituto de Geología, de la Universidad Nacional Autónoma de México, por lo que a los estudiantes que facilitaron sus materiales les expresamos nuestro agradecimiento.

\section{EL INICIO DE LA HISTORIA}

Con relación a las ideas expresadas en la introducción es claro que los procesos biológicos que se asocian a la generación de distintas formas de vida han existi- 
do cuando menos durante los últimos 3700 millones de años. La pregunta entonces es qué hace que los procesos se detonen y funcionen en diferentes momentos. Aquí es en donde la geología juega un papel importante. Los estudios geológicos han ayudado a comprender cómo la formación de los primeros continentes y su modificación tanto en configuración como relieve debido a procesos tectónicos y/o volcánicos, conllevan variación geográfica a lo largo del tiempo geológico. El espacio de lo que hoy conocemos como México no ha sido ajeno a estos procesos y la vida que se desarrolla sobre la parte continental emergida ha tenido que afrontar y adaptarse a las condiciones fisiográficas que moldearon el entorno de manera diferente a lo largo de la evolución geológica.

Se han documentado plantas que habitaron en México durante el Triásico (Sonora) y Jurásico (Chiapas, Oaxaca, Puebla, Veracruz, Hidalgo) (e.g., Silva-Pineda y Buitrón-Sánchez, 1999; Weber, 2008a,b,c). Sin embargo, estas plantas en su mayoría son extintas en la actualidad y solo algunos representantes de estos linajes continúan creciendo en ciertas zonas de México, como es el caso de las cícadas o las coníferas. Iniciamos entonces la revisión en el Cretácico cuando aparecen las flores y frutos, los colores se multiplican y los olores llenan el ambiente, todo esto proponiendo una nueva biología, formas nuevas de interacción, de dispersión, de colonización, etc. Este inicio refleja un momento con gran impacto en la vida que puede equipararse con el origen de la vida, la aparición del sexo, la colonización de las partes emergidas, la administración de la dependencia del agua a través de la semilla y el huevo amnioto, etc. En todos estos momentos el desarrollo de innovaciones morfológicas, anatómicas y fisiológicas es importante, pero lo que realmente revoluciona a la vida es la forma en que las novedades biológicas se integran al sistema en el que la vida se desarrolla.

\section{LA HISTORIA E HIPÓTESIS}

Cambios paleogeográficos y evolución de la flora

\section{El Cretácico}

Es importante iniciar señalando que durante el Cretácico la temperatura global fue muy elevada por las altas concentraciones de $\mathrm{CO}_{2}$, causando un gradiente latitudinal bajo lo que permitió el establecimiento de floras termófilas en las latitudes medias y altas. En medio de este escenario, durante el Cretácico (144-65 ma) prácticamente todo el país estuvo cubierto por mares (Goldhammer, 1999; Eguiluz 
de Antuñano et al., 2000), impidiendo que muchos de los linajes que habitaban las zonas que se cubrieron por mares continuaran desarrollándose en esas regiones. Aunque existieron islas donde la vida continuó, estos "relictos" estuvieron sujetos a fuertes presiones de selección, resultando que la gran mayoría de las plantas que hoy crecen en México están relacionadas con linajes que fueron estableciéndose en regiones emergidas conforme los mares se retiraron, a partir del Cretácico (Fig. 1).

Este fenómeno de regresión de los mares sucedió de manera general siguiendo un desplazamiento hacia el este y sureste. Se inició en el Cretácico y continuó durante el Cenozoico (65-1.8 ma), y durante gran parte de este tiempo América del Norte y del Sur estuvieron separadas, aunque entre ellas existían algunas islas que bien pudieron servir de sitios que facilitaron la ampliación de la distribución de algunos linajes, tanto de aquellos establecidos en el norte extendiéndose hacia el sur, como en dirección contraria. La evidencia con la que contamos sugiere que las plantas de latitudes más altas de América del Norte acrecentaron su distribución hacia el límite del continente estableciéndose en lo que en aquel tiempo era una proyección de las partes emergidas, formando una península conformada por algunas porciones de los estados de Tamaulipas, Nuevo León, Coahuila, Chihuahua, Sonora y Baja California (Fig. 1). Aunque queda mucho por documentar, se sabe que crecieron abundantes Arecaceae con hojas pinnadas y quilladas, representantes de Zingiberales, probables, Fagaceae, Burseraceae y/o Anacardiaceae, desde luego miembros de Laurales, y de Euphorbiaceae, además de miembros de Pinaceae, Cheirolepidaceae y probablemente Taxodiaceae eran compartidas entre la costa y la parte central de América del Norte (e.g., Rodríguez de la Rosa y Cevallos-Ferriz, 1994; Rodríguez de la Rosa y Cevallos-Ferriz et al., 1998; Hernández-Castillo y Cevallos-Ferriz, 1999; Calvillo-Canadell y Cevallos-Ferriz, 2007; Estrada-Ruiz et al., 2010) (Fig. 2 y 3).

América del Norte durante el Cretácico Superior (99-65 ma) estuvo dividida por un mar epicontinental, el Mar Interior del Oeste ("Western Interior Seaway"), comprendido entre la zona alrededor de la frontera Coahuila-Tamaulipas/Nuevo México-Texas-Luisiana en el NE de México (en donde después se forma el Golfo de México) y hasta el Océano Ártico (al este de la Península de Alaska). Las porciones emergidas estaban divididas en dos regiones que contienen registros polínicos contrastantes, la provincia de los Normapolenes al este que se extiende hacia Europa, y la provincia de Aquillepolenites al oeste, que se extiende hacia Asia. En México los macrofósiles de la parte este se colectan en localidades de la región costera del mar epicontinental (Coahuila, Tamaulipas), mientras que al oeste hay localidades cercanas al Océano Pacífico (Baja California), y en la región continental entre los mares 

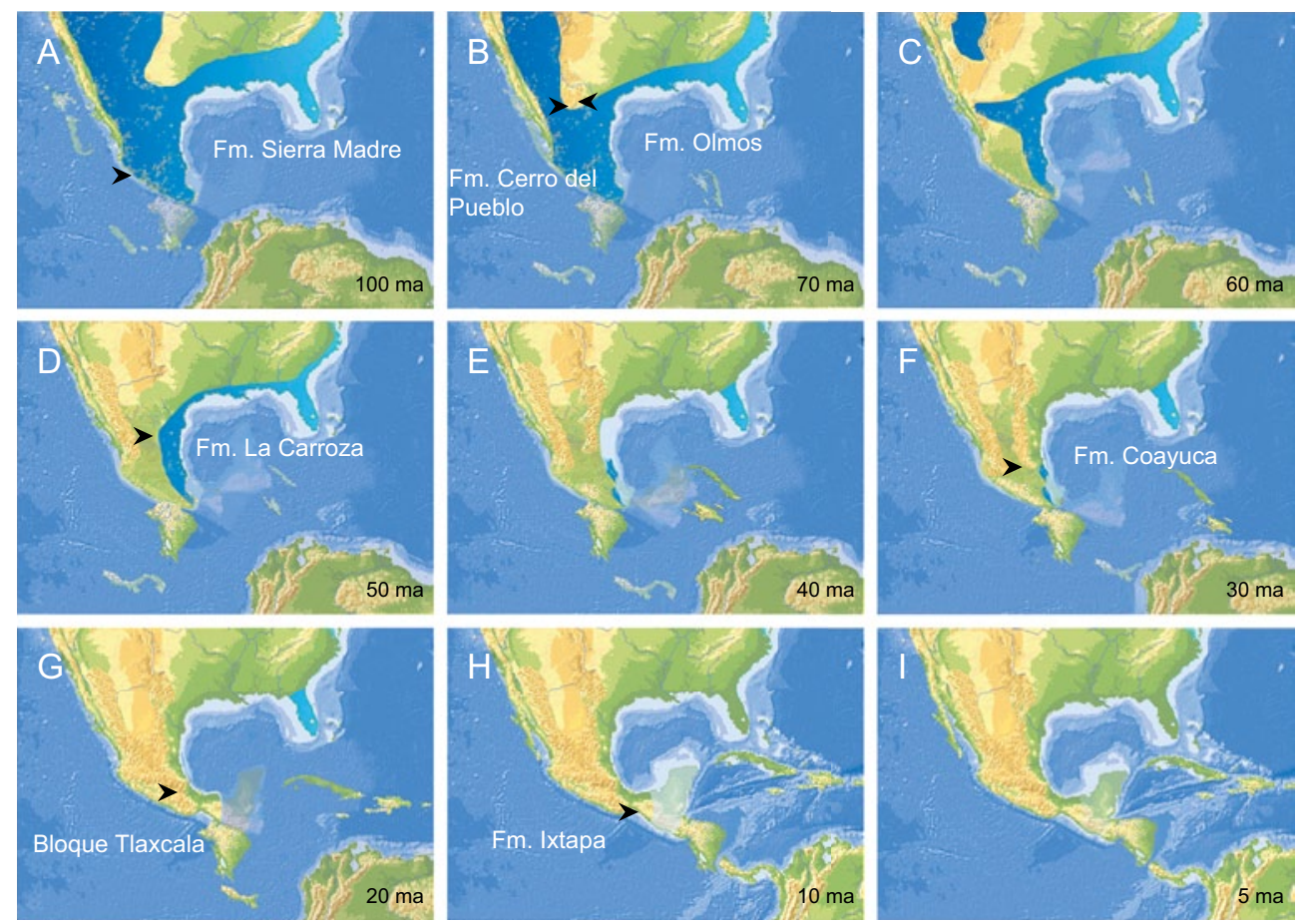

Fig. 1. Diagramas representando de forma muy general la evolución geológica de México. A. transgresiones marinas generalizadas; B. levantamiento de la porción noroeste; C. continúan regresiones y está en proceso la deformación Laramídica; D. actividad volcánica importante en extensión; E. desplazamiento del bloque Chortis y arco volcánico muy ancho; F. paroxismo ignimbrítico en el (ca. 32-28 ma) W; G. pulsos de extensión (ca. 24-20 ma) y cambio de orientación del arco volcánico (25-17ma); H. inicio del proceso de apertura del Golfo de México y formación de la Faja Volcánica Transmexicana; I. emersión de la Península de Yucatán y termina conexión entre el Pacífico y Caribe.

mencionados (Sonora). En conjunto los fósiles sugieren que las comunidades aunque compuestas de gimnospermas y angiospermas, diferían en la dominancia de estos grupos, siendo las plantas con flor más abundantes y comunes en la parte oriental (e.g., Cevallos-Ferriz, 1983; Cevallos-Ferriz y Weber, 1992; Hernández-Castillo y Cevallos-Ferriz, 1999; Estrada-Ruiz et al., 2008, 2011). La vegetación que se ha podido documentar habitando esta península recuerda a selvas con poca estacionalidad, con abundantes cuerpos de agua y altas temperaturas. Particularmente en Coahuila se postula la presencia de una selva paratropical, posiblemente la primera de este tipo en América del Norte (Estrada-Ruiz et al., 2011). En contraste, la vege- 

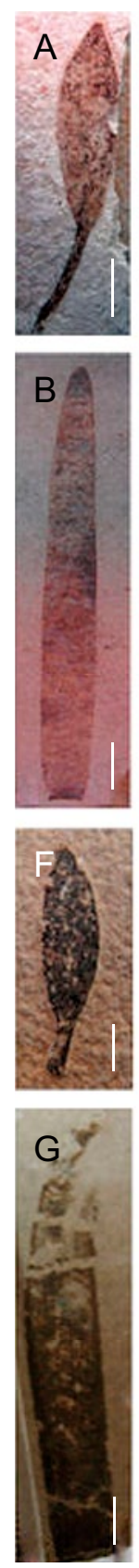
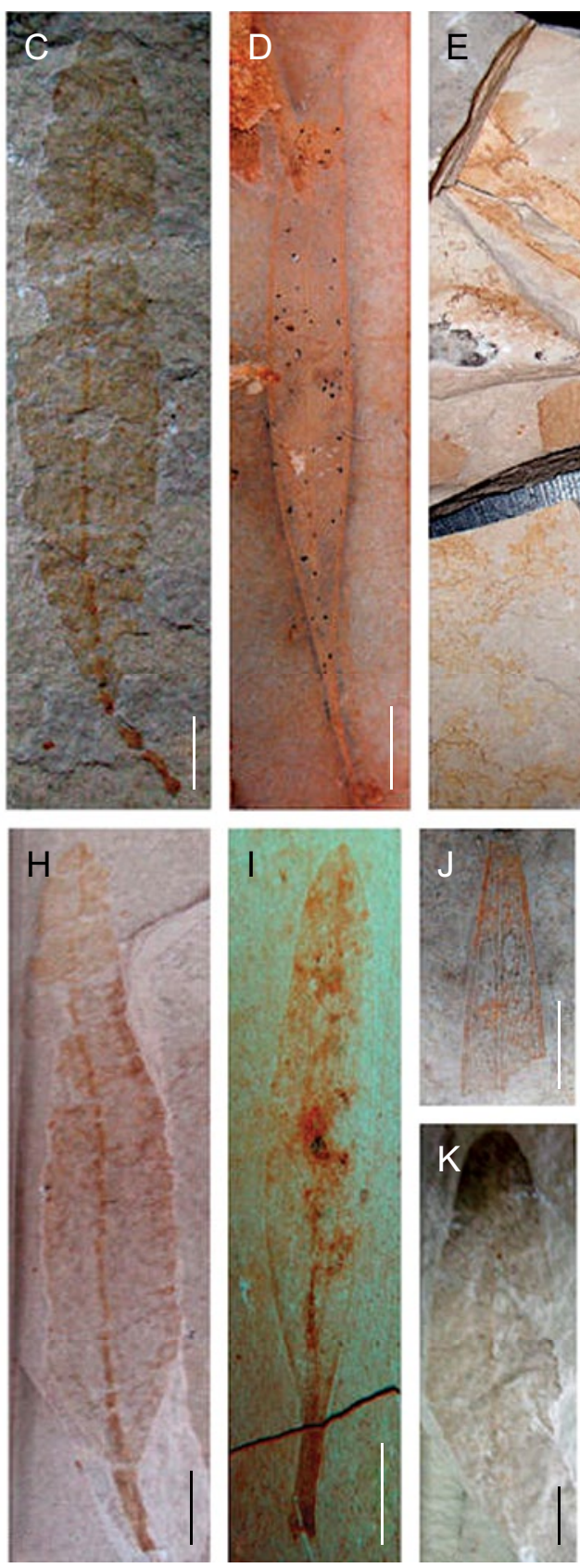
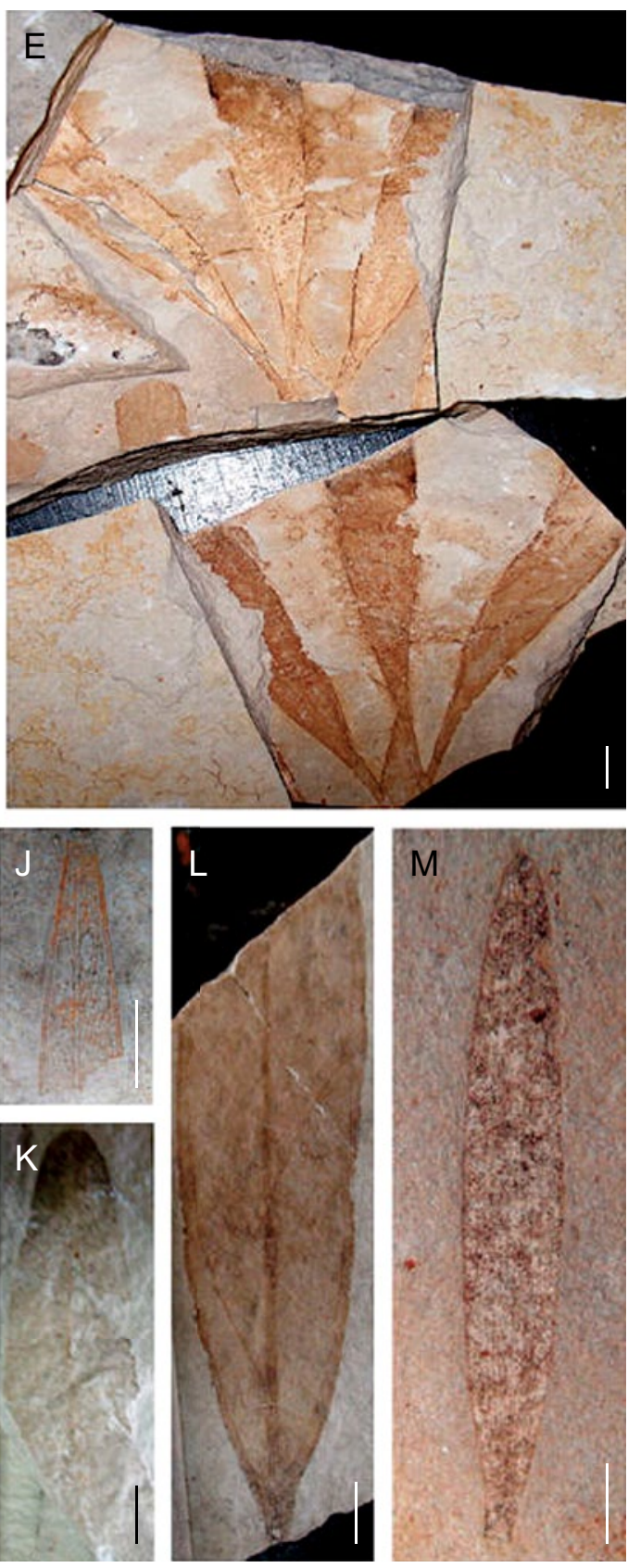

Fig. 2. Angiospermas cretácicas de la localidad del Chango, Chiapas. Material cortesía de Griselda Guerreo Márquez. Escala $=1 \mathrm{~cm}$. 
Cevallos-Ferriz et al.: Paleobotánica y biodiversidad en México
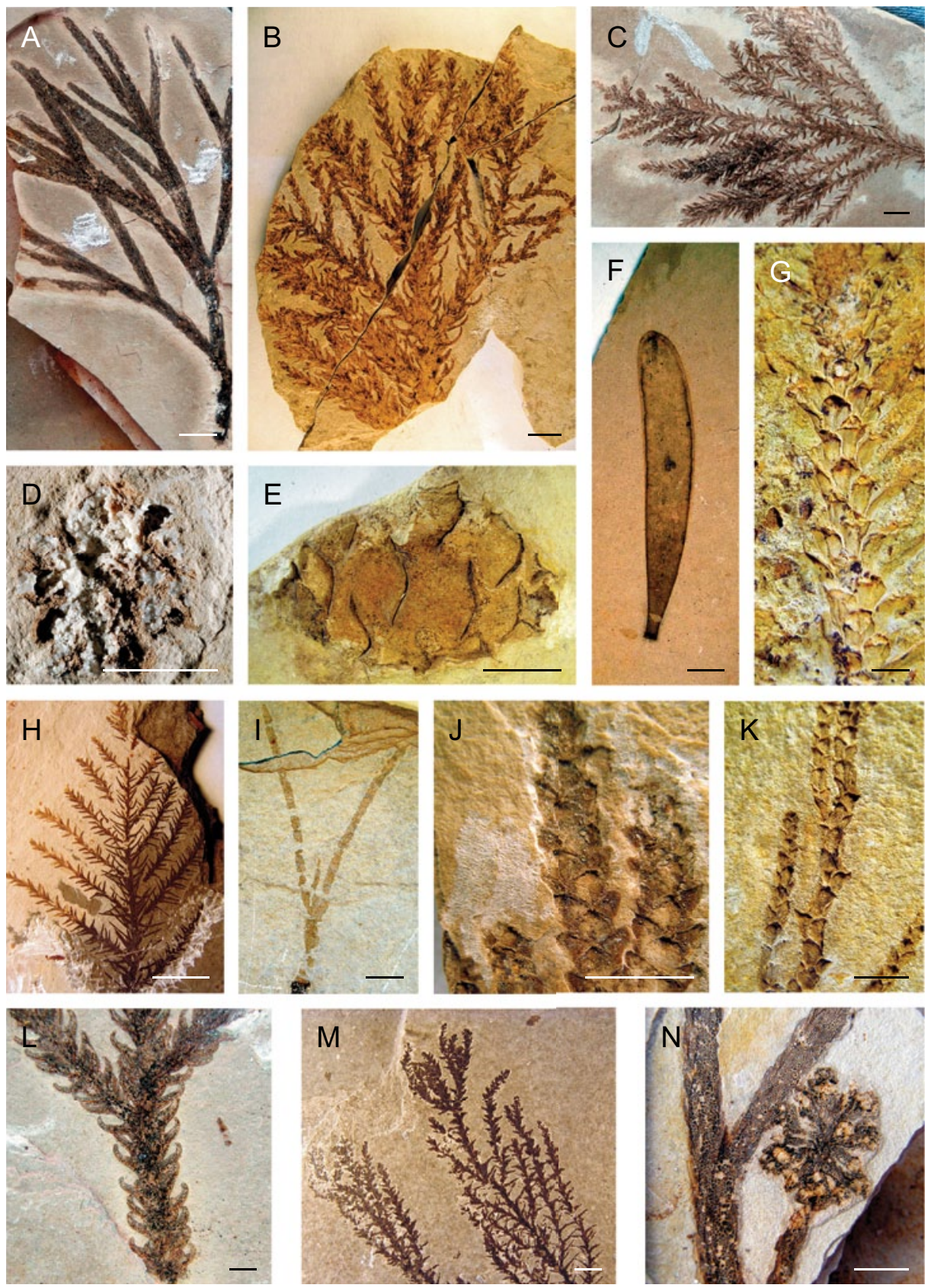

Fig. 3. Gimnospermas cretácicas de la localidad del Chango, Chiapas. Material cortesía de Ixchel González y Alma R. Huerta. Escala $=1 \mathrm{~cm}$. 
tación hacia el oeste parece corresponder a clima más templado y por lo mismo las coníferas son más comunes (Cevallos-Ferriz, 1983; Cevallos-Ferriz y Weber, 1992). Las elevaciones hacia el centro y occidente de esta región probablemente generaron diferencias en la composición de las comunidades de aquel momento, facilitando que plantas propias de clima más fresco se establecieran (Figs. 2 y 3).

En el sur de México hasta hace relativamente poco tiempo no se habían recolectado plantas del Cretácico. La presencia ocasional de coníferas había sido reportada anteriormente por paleontólogos que trabajan peces e invertebrados de la Formación Sierra Madre. Durante una prospección para constatar estos reportes, se confirmó la abundancia de ramas de coníferas y conos asociados, algunos de ellos en conexión orgánica; pero de manera inesperada, se recolectaron cuando menos unos 20 morfotipos de órganos de plantas con flor, que incluyen frutos y hojas. Comparando este material con las plantas conocidas del norte de México, es sorprendente que aunque sí hay Pinaceae, aparentemente son más abundantes las Podocarpaceae, Araucariaceae y Cupressaceae; además, hasta el momento solo puede documentarse que comparten un tipo de planta con flor representado por sus hojas, Manihotites (Euphorbiaceae), cuyo tamaño es claramente distinto. Otros registros de angiospermas del sur de México, en específico Chiapas, incluyen pastos marinos, una hoja semejante a Sapindites y varios frutos cuya afinidad es un enigma en este momento (e.g., González-Ramírez et al., 2011; Guerrero-Márquez et al., 2011; Huerta-Vergara et al., 2011) (Figs. 2 y 3). Posiblemente las diferencias en floras entre el norte y sur de México se deban, como sugieren los análisis palinológicos del centro y sur del país, a la presencia de elementos que corresponden con las provincias de Monocolpates y Nothofagidites, del norte de América del Sur.

Aunque falta mucho para realmente poder comparar de manera completa las floras del norte y sur de México, tienen un aspecto en común, consistente en que las plantas, o los órganos de estas, sugieren que en el Cretácico vivían muchas plantas que pueden relacionarse con alguna familia, y aún compararse con géneros actuales, pero que no se pueden incluir en ellos. Aún más, la reconstrucción de plantas, es decir las propuestas de que dos o más órganos representan a una misma planta, sugieren que muchos de estos taxa pudieran ser parte del grupo troncal ("stem") del clado que representan. La supuesta asociación de los troncos con madera tipo Paraphyllanthoxylon con hojas de Anacardiaceae por un lado y Lauraceae por otro, son un buen ejemplo de esto (Cevallos-Ferriz, 1983; Méndez-Cárdenas et al., sometido). Ninguno de estos órganos, aunque pueden relacionarse con estas familias, tienen canales o idioblastos; elementos comunes entre sus representantes actuales. Lo mismo sucede con las maderas que parecen ser miembros de Fagaceae, su construcción 
recuerda en cierta forma a los encinos, pero siempre hay características que los separan (Estrada-Ruiz et al., 2010, 2011). Es igualmente interesante la propuesta de que existieron plantas acuáticas parecidas a grupos actuales como Nuphar o Salvinia, pero la comparación morfológica detallada apoya que se trata de taxa extintos, a veces también reportados de otras partes del mundo (Estrada-Ruiz et al., 2011). La presencia de grupos como Musaceae y Zingiberaceae llaman la atención pues su área de distribución natural cambia radicalmente del Cretácico al Neógeno (Rodríguez de la Rosa y Cevallos-Ferriz, 1994). Ahora parece que se debe añadir otro grupo que también cambia su distribución natural al avanzar el tiempo; las Proteales están representadas en el Cretácico de México por hojas, polen y frutos, aunque por el momento los órganos aislados se conocen de diferentes localidades (GuerreroMárquez et al., 2011; Vázquez-Rueda et al., 2011).

Seguramente en las partes emergidas de México existían sistemas de montañas que hicieron variar el relieve y las condiciones ambientales locales donde se establecieron las plantas. Las elevaciones en ese tiempo eran aparentemente menos importantes que las cadenas montañosas que se desarrollaron al occidente y oriente conforme se continuaba retirando el mar hacia el sur durante el Cenozoico, pero seguramente tuvieron gran influencia para generar escenarios distintos en los que se establecieron comunidades diferentes a las que hoy conocemos (Fig. 1). Las variaciones ambientales de tipo climático de aquel tiempo no necesariamente se comparan con alguna situación actual, pues durante ese periodo la temperatura alcanzó un máximo que seguramente influyó en la distribución latitudinal de la paleoflora.

Cambios en los escenarios durante la transición Cretácico/Cenozoico modificaron las condiciones ecológicas sobre el continente. Fueron importantes las regresiones del mar epicontinental que dividió América del Norte durante el Cretácico. $\mathrm{Su}$ retiro hace más clara la presencia de la "península cretácica" del sector sur de América del Norte, en donde se encontraba una porción de México, que continuó creciendo hacia el sur, y sobre las superficies que se venían exponiendo se desarrollaron elevaciones importantes (Cevallos-Ferriz y González-Torres, 2005). Esta visión muy simplificada de la evolución geológica de México permite postular la presencia de una parte central limitada por montañas cada vez más importantes, que a su vez generaban planicies costeras con distintas características al oeste y este del país (Fig. 1).

Un segundo gran cambio que afectó a la vida en el Cretácico y su transición al Cenozoico fue producido por el impacto del meteorito Chicxulub (Urrutia-Fucugauchi, 2011), que alteró de manera abrupta las condiciones ambientales y jugó un papel importante en someter y poner a prueba las capacidades biológicas de las 
especies de ese tiempo a las exigencias drásticas y momentáneas que generó. Esta región de América del Norte debió de haber sido sometida a cambios aún mayores por la cercanía del impacto y la fuerza de los efectos secundarios en las regiones vecinas al mismo.

Sin embargo, el desarrollo con dirección norte-sur de la Sierra Madre Occidental, cuya configuración inició en el Cretácico tardío, terminando en el Mioceno medio (Ferrari et al., 2005), y de la Sierra Madre Oriental también iniciando en el Cretácico/Paleoceno y terminando en el Mioceno (Eguiluz de Antuñano et al., 2000), producen importantes cambios fisiográficos en la región.

Estas modificaciones del entorno abiótico debieron de haber repercutido en las comunidades que habitaban el sur de América del Norte, y seguramente fueron importantes en la selección de especies que formaron parte de las comunidades que fueron ocupando los nuevos escenarios que se iban integrando. Tales procesos suceden en un corto tiempo. La interacción entre los cambios en los escenarios y las respuestas biológicas ha sido continua desde el Cretácico, siendo la acumulación de las respuestas exitosas a las persistentes pruebas del medio promovidas por los cambios constantes, lo que ha hecho que se acumule la diversidad.

\section{Cenozoico}

Aunque son pocas las paleofloras que conocemos del Paleógeno de México (Paleoceno (65-55 ma) - Eoceno (55-33 ma) - Oligoceno (33-23 ma)), la flora de la Formación Carroza (Eoceno Medio) ejemplifica cómo para este tiempo las plantas con hojas simples, márgenes enteros y talla grande comunes en el Cretácico son sustituidas por otras en las que las hojas pinnadas con márgenes serrados, dentados o crenados y talla menor eran más comunes (Calvillo-Canadell y Cevallos-Ferriz, 2005; Calvillo-Canadell et al., sometido). El cambio en los patrones morfológicos de las hojas sugiere modificaciones en el ambiente que obviamente seleccionaron a las plantas que vivían en distintas localidades. En la misma Formación Carroza se han recolectado hojas de mayor tamaño, aunque no alcanzan la talla de las cretácicas; pero estas son contenidas en lutitas de grano, textura y color distinto a las que contienen a las hojas de menor talla. Tanto las características de los sedimentos, como de las hojas que contienen, sugieren que en esta localidad se acumularon plantas de dos regiones distintas, aunque geográficamente cercanas, en las que las condiciones ambientales locales permitieron el establecimiento de comunidades parcialmente diferentes, una más xerófila (Soeegard et al., 2003), con hojas pequeñas y dentadas, y otra más mesófila, con plantas con hojas de mayor tamaño y mayor número de hojas enteras (Rodríguez-Reyes, 2007). El polen reportado para el noreste del país incluye 
también una mezcla de grupos que son más típicos de vegetación cálido-húmeda y al mismo tiempo contiene elementos de fitocenosis que se desarrollan a menor temperatura y humedad (Figs. 1, 4).

Debe resaltarse también que el registro de macrofósiles en el norte del país no contiene coníferas de esta edad, contrastando con su importancia durante el Cretácico, sin embargo, polen de este grupo sí se reporta. Aunque las microsporas de Pinus y Picea se señalan como presentes, macrofósiles de estos géneros no se han recolectado; las coníferas anteriores son taxa extintos relacionados con la misma familia. En el sur de México tampoco se han recolectado macrofósiles pero es interesante que además de polen de Pinus, otros como los de Picea y Larix también se han reconocido. Llama la atención que polen de plantas "exóticas" para el país, como Keteleria, siguen estando presentes durante el Mioceno. Este material "exótico" aparentemente puede trazarse en la región al Cretácico, así es que debieron extinguirse de esta parte sureña de América del Norte en algún momento post-oligocénico (Palacios Chávez y Rzedowski, 1993; Martínez-Hernández y Ramírez-Arriaga, 1996).

Para el norte del país esta situación puede explicarse si se consideran los procesos geológicos que estaban alterando la fisiografía de la región, convirtiendo a los estados de Coahuila y Nuevo León, en una zona de mayor continentalidad, al estar localizadas en la porción occidental de la Sierra Madre Oriental, en proceso de formación durante el Neógeno. Tal elevación topográfica aleja a Coahuila y Nuevo León de la región del Golfo de México, además la humedad se ve restringida por el retiro de los mares epicontinentales. Entonces, a partir del Paleógeno la evolución geológica y fisiográfica del norte de México, y la tendencia global de enfriamiento que inicia en el Eoceno medio, acentúan el desarrollo de zonas con regímenes anuales no constantes de humedad y temperatura. Es decir, la estacionalidad aparentemente se hace presente y su intensidad seguramente varió de acuerdo con las localidades. Con esto no se sugiere que ya existieran zonas áridas como se conciben hoy, pero sí es claro que las condiciones para que estas zonas se establecieran y expandieran iniciaron en esta época de importante actividad tectónica en la región norte del país. Posiblemente la presencia de estacionalidad cada vez más marcada en distintos lugares impulsó la selección biológica de las poblaciones que habitaban en la región, generando nuevas agrupaciones de plantas que respondían a las nuevas exigencias del medio físico, aunque seguramente persistieron en otras zonas las comunidades originales. Aún más, el norte del país sufrió a lo largo del Paleógeno diferentes niveles de elevación que contribuyeron a generar muy diversos escenarios en los que las plantas se agruparon formando comunidades distintas. La presencia de Bursera, un elemento típico de las selvas bajas actuales, en la Formación La Carroza, junto 
con plantas relacionadas con Myrtaceae (cf. Eugenia) o Leguminosae (Inga, Senna, Chamaecrista) sugieren que esta interpretación es muy probable, pero debe seguir documentándose (Calvillo-Canadell y Cevallos-Ferriz, 2005; Rodríguez-Reyes, 2007; Calvillo-Canadell et al., sometido) (Fig. 4).

Continuando con la expansión y desarrollo de los tipos de vegetación más xerófilos en México, los fósiles sugieren que para el Oligoceno (33-23 ma) en el centro de México ya se habían establecido comunidades similares a las selvas bajas o chaparrales. La flora de los Ahuehuetes, de la Formación Coatzingo, cerca de Tepexi de Rodríguez, Puebla, es un ejemplo de un proceso que aparentemente inició en el norte (e.g., Fm. Carroza) y alcanza el centro de México hace aproximadamente 32 millones de años (Calvillo-Canadell y Cevallos-Ferriz, 2002, 2005; Calvillo-Canadell et al., sometido). Las hojas que se han recolectado de esta región son aún más pequeñas que las del Eoceno de La Popa y las hojas compuestas son más comunes. Entre los representantes hay formas que actualmente son endémicas de zonas secas en Perú y Bolivia, como Haplorhus (Anacardiaceae). Además de otras que pueden indicar cierto grado de estacionalidad como Rhus, Pistacia, Comocladia (Anacardiaceae), o miembros de Leguminosae como Inga, Pithecellobium, Mimosa, Sophora, Cladrastis y Apuleia entre otras y desde luego miembros de Berberidaceae entre los que se han identificado a Berberis y Mahonia, a veces incluidos en el mismo género (Calvillo-Canadell y Cevallos-Ferriz, 2002, 2005). Pero también hay elementos que pueden establecerse en zonas con mayor humedad, como las Rosaceae y otros posiblemente representando elementos riparios como las Salicaceae (Salix y Populus). Aún más, las maderas de otra localidad, Coayuca de Andrade, Puebla, presumiblemente de la misma edad que las plantas de los Ahuehuetes, se caracterizan por representar plantas con anillos de crecimiento bien marcados, sugiriendo estacionalidad acentuada en la región y entre las que se han identificado Anacardiaceae y Leguminosae (Magallón-Puebla y Cevallos-Ferriz, 1994a,b,c; Ramírez et al., 2000; Ramírez y Cevallos-Ferriz, 2000a,b, 2002; Velasco de León y Cevallos-Ferriz, 2000). Otra localidad del Oligoceno de Puebla, cerca de San Juan Atzingo contiene maderas entre las que se han identificado Leguminosae (Prioria y Dalbergia) y Boraginaceae (Cordia), que continúan apoyando el establecimiento de vegetación semejante a selvas bajas en la región hace ca. 32 millones de años (Sainz-Reséndiz, 2011).

La expansión de floras más xerófilas al centro y sur de México parece seguir otra serie de procesos geológicos significativos relacionados con importantes cambios hidrológicos que secaron una serie de cuencas lacustres. Estas transformaciones pueden ser inducidas o acompañadas por el proceso de elevación de la parte centro y 
Cevallos-Ferriz et al.: Paleobotánica y biodiversidad en México
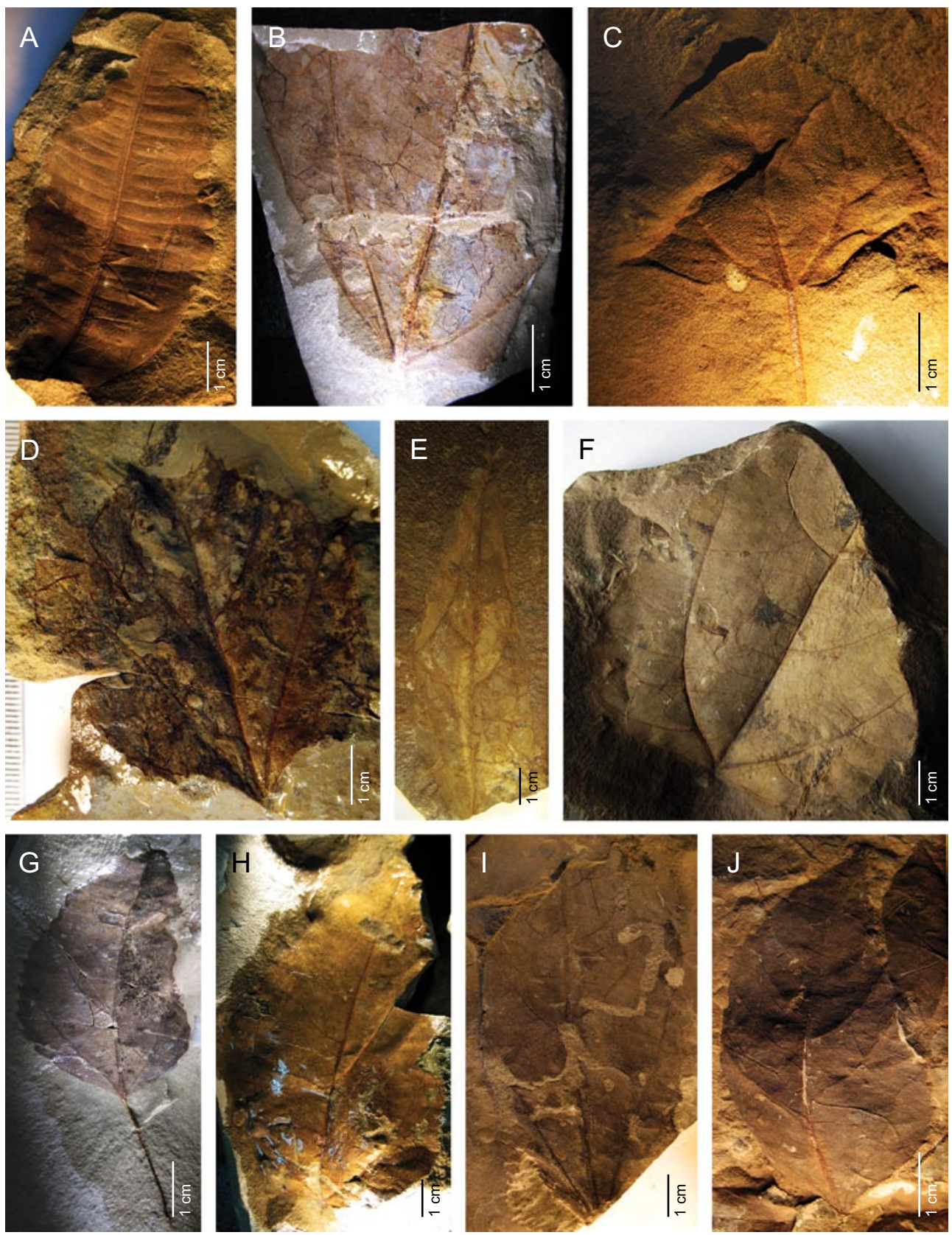

Fig. 4. Hojas de la Formación la Carroza, La Popa, Nuevo León, del Eoceno medio. Material cortesía de Ana Isabel Pérez Maussán. 
sur de México a partir del Oligoceno/Mioceno debido al cambio en la geometría de la Sierra Madre Occidental y formación de la Faja Volcánica Transmexicana (Ferrari et al., 1999). Esta terminó aislando a la porción central norte de México, ayudando a restringir más la captación de agua de la región, pero a la vez contribuyó a mantener y/o incrementar las zonas húmedas del sur, promoviendo vegetación con menor estacionalidad y hojas de mayor tamaño.

Sin embargo, es importante mencionar que con base en los macrofósiles la primera comunidad con elementos típicamente mesófilos se encuentra en el Plioceno de Santa María Amaxac, Hidalgo. En esta localidad se reportan plantas como Platanus, Quercus, Populus, etc., sugiriendo que este tipo de vegetación también se ve favorecido por los diversos escenarios que se produjeron al formarse la Faja Volcánica Mexicana (Gómez Tuena et al., 2005; Velasco-de León y Ortiz-Martínez, 2010), que dio origen a importantes elevaciones sobre el nivel del mar. Junto a la evidencia con macrofósiles hay que añadir que los estudios palinológicos reconocen a varios miembros de plantas que pudieron desarrollarse en distintos tipos de bosques mesófilos a partir de sedimentos tan antiguos como el Oligoceno (MartínezHernández y Ramírez-Arriaga, 1996). La combinación aparentemente poco común de plantas, con base en un marco de referencia actual, sobre todo en el Paleógeno y la separación de estas en tipos de vegetación cada vez más parecidos a los actuales durante el Neógeno posiblemente tiene que ver con la desintegración de la Flora Boreotropical conforme la fisiografía del territorio nacional ofreció a las plantas la posibilidad de establecerse en distintos escenarios, en donde cada vez fueron más seleccionadas las asociaciones típicas que hoy se reconocen.

Las plantas del trópico húmedo que caracterizaron al norte de México durante el Cretácico, no se han recolectado de forma clara en otros sitios del país en rocas más jóvenes, posiblemente porque estas comunidades crecieron a lo largo de las costas del oriente y occidente, y las localidades fosilíferas estudiadas hasta el momento se encuentran hacia el centro del país. Sin embargo, la flora de Baja California Sur, de la Formación El Cien, del Mioceno, contiene maderas de plantas que sugieren que en aquel momento en la región estaba establecida una flora que taxonómicamente recuerda a la selva baja de Chamela, Jalisco, pero un estudio de anatomía ecológica indica que la humedad y temperaturas eran elevadas, seleccionando el establecimiento de una selva alta (Martínez-Cabrera et al., 2006; MartínezCabrera y Cevallos-Ferriz, 2006). Entre las plantas reconocidas de esta localidad aunque no se han estudiado con detalle se encuentran Ficus, Maclura (Moraceae), Andiroxylon, Mimosoxylon (Leguminosae) y Tapirira (Anacardiaceae). En el Istmo de Tehuantepec, tanto en Veracruz, como en Oaxaca, se han recolectado floras del 
Mioceno que sugieren que este tipo de comunidades se extendía hacia el sur. Los fósiles del Oligoceno/Mioceno de Simojovel, Chiapas (Fig. 5), apuntan que tales floras del trópico húmedo efectivamente continuaron desarrollándose a lo largo de las costas (Miranda, 1963; Castañeda-Posadas y Cevallos-Ferriz, 2007; Padi1la, 2007; Calvillo-Canadell et al., 2010; Cevallos-Ferrizy Calvillo-Canadell, 2010) (Fig. 6). Recientemente se ha recolectado otra flora del Mioceno en la Formación Ixtapa, Chiapas, que por el tamaño de sus hojas y el reconocimiento de algunos taxa, se propone que representa otra comunidad propia de clima cálido húmedo. No obstante, un análisis paleoclimático basado en arquitectura foliar sugiere que las plantas crecían bajo condiciones que más bien favorecerían el establecimiento de bosques mesófilos (Peralta-Medina, 2009; Domínguez de la Torre et al., 2011) (Figs. 7, 8). Entonces, en Chiapas, durante el Oligoceno/Mioceno como en el Eoceno de Nuevo León, se detectan floras semejantes bajo condiciones climáticas distintas. Con base en los fósiles del ámbar de Simojovel de Allende, Chiapas, se documenta la presencia de una selva tropical, aspecto que contrasta con la flora de Ixtapa, Chiapas, que aunque aparentemente contenía plantas que podían encontrarse en una región cálido-húmeda, el análisis paleoclimático sugiere que crecieron bajo condiciones más frías; no obstante, ambas se desarrollaron al nivel del mar o muy cerca de este (Figs. 7, 8).

Bajo esta perspectiva las zonas áridas (“desiertos” actuales) de México parecen tener una historia relativamente corta, aunque sus componentes pueden trazarse hasta el Paleógeno, como lo señala la presencia de Bursera y algunas leguminosas en el Eoceno de La Popa, Nuevo León, o las Berberidaceae, otras leguminosas y anacardiáceas del Oligoceno de Los Ahuehuetes, Puebla (Magallón Puebla y Cevallos-Ferriz, 1994b; Ramírez y Cevallos-Ferriz, 2000a,b; Calvillo-Canadell y Cevallos-Ferriz, 2002, 2005).

El registro de los macrofósiles muestra entonces que aparentemente los linajes de los distintos tipos de vegetación que hoy reconocemos en el país pueden trazarse cuando menos desde el Paleógeno (Eoceno-Oligoceno), no tenemos aún registro del Paleoceno, y a la vez sugieren que aunque las comunidades del pasado tengan semejanza taxonómica con alguna comunidad actual, siempre hay diferencias. Por ejemplo, en las fitocenosis del pasado se detectan taxa que ya se extinguieron o no viven más en México o la región geográfica implicada, no permitiendo una comparación uno a uno entre las paleocomunidades y las neocomunidades. Pero además, estudios detallados de morfología comparada de las plantas pre miocénicas con las actuales han demostrado que la relación taxonómica de las que crecieron en México en aquellos tiempos era mayor con las que crecieron en latitudes 

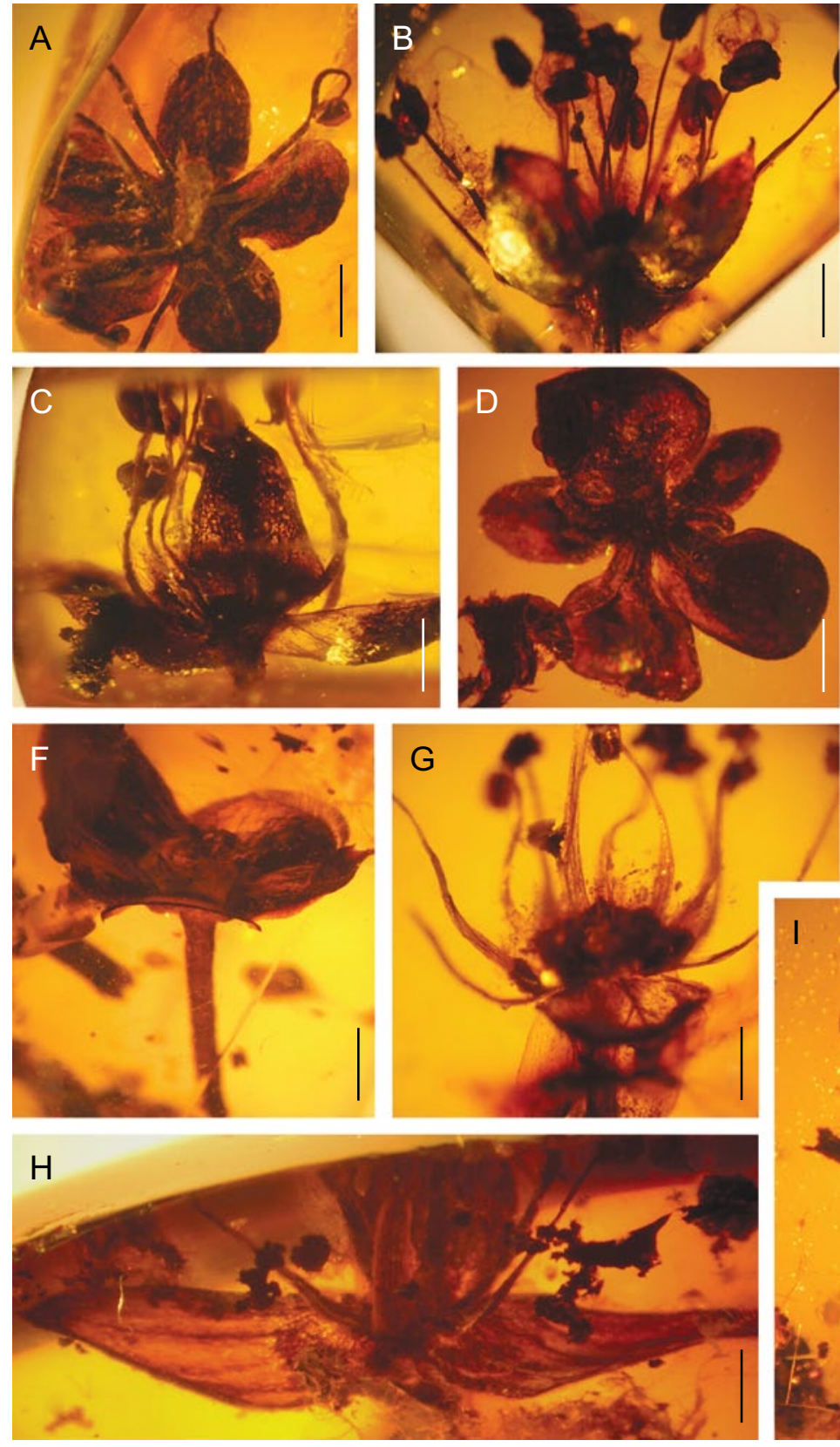

Fig. 5. Flores conservadas en el ámbar del Oligoceno/Mioceno de Simojovel de Allende, Chiapas. Material cortesía de Ana Lilia Hernández Damián. Escala $=1 \mathrm{~cm}$. 

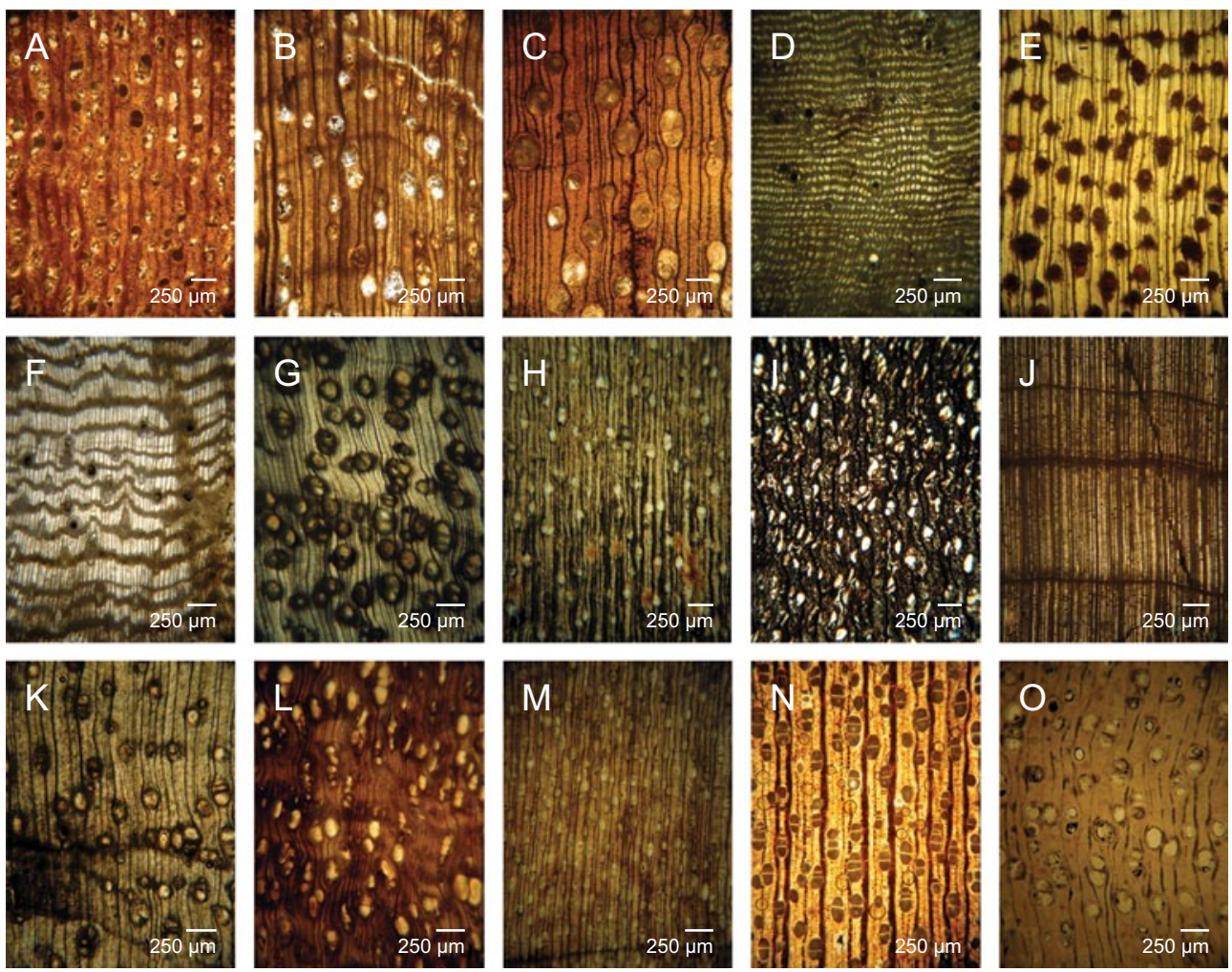

Fig. 6. Maderas del Oligoceno de Puebla. Mioceno de Tlaxcala y Chipas y Plioceno de Culiacán. Material cortesía de Luis A. Flores R. y Juliana Cárdenas.

mayores del Hemisferio Norte. Esto genera la pregunta de ¿cuándo se "modernizan” las plantas que crecen en México? o ¿cuándo las plantas fósiles recolectadas en México muestran mayor afinidad con las que crecen actualmente en el país y se diferencian de aquellas pre miocénicas? Las respuestas no la conocemos bien, pero la evidencia sugiere que es a partir del Mioceno que la cercanía morfológica entra taxa fósiles y actuales es mayor, seguramente señalando afinidad taxonómica más cercana. Esta "modernización" va muy ligada con la dinámica de formación de los cinturones montañosos de las Sierras Madre Occidental y Oriental y la gran actividad de la Faja Volcánica Transmexicana, dando lugar a procesos geológicos generadores de cambios altitudinales, climáticos y muy importantes en la diversificación y radiación de taxa que pudieron establecerse en sitios con características variadas (Gómez Tuena et al., 2005). 

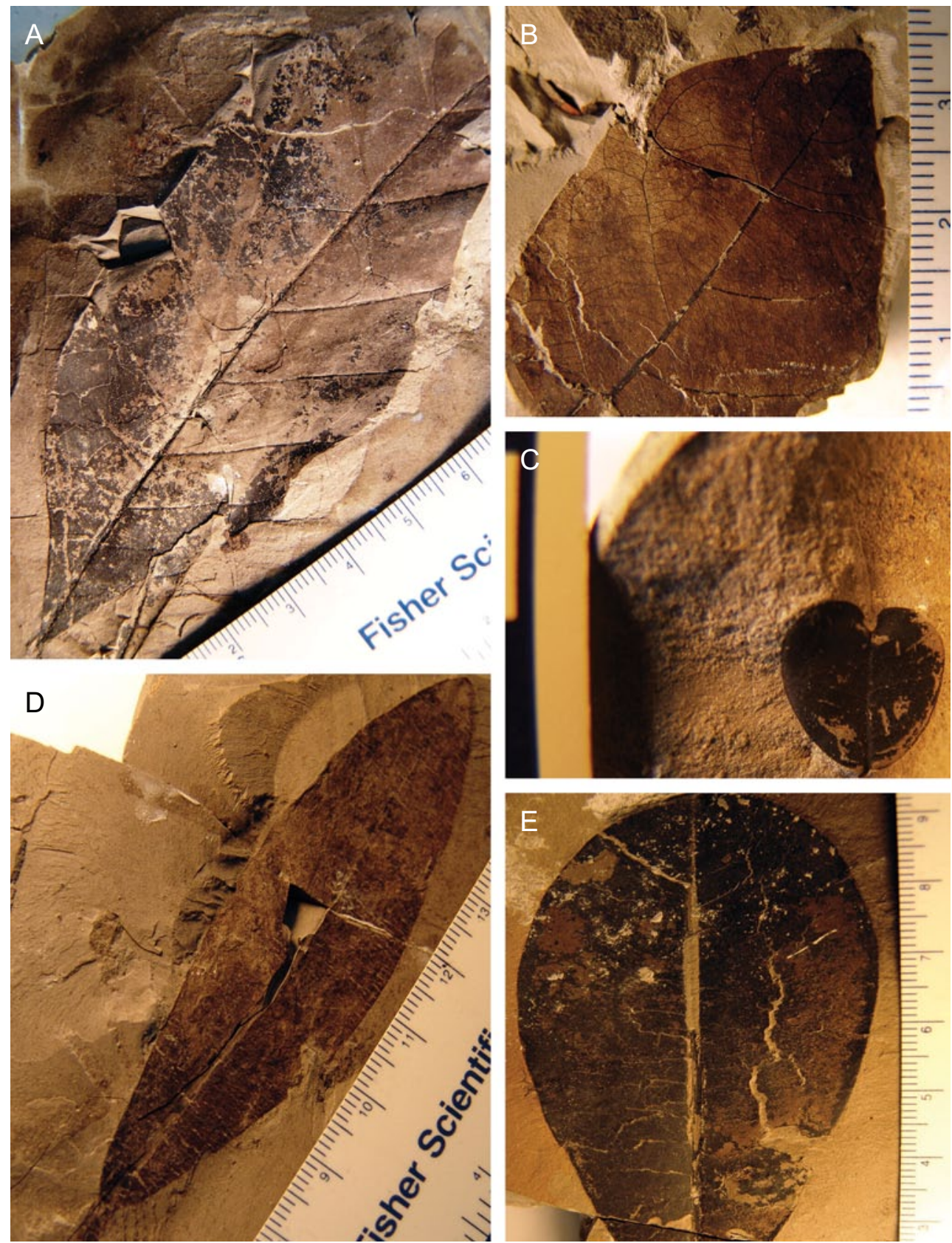

Fig. 7. Hojas del Mioceno de Ixtapa, Chiapas. Material cortesía de Aldo Domínguez de la Torre. 
Cevallos-Ferriz et al.: Paleobotánica y biodiversidad en México
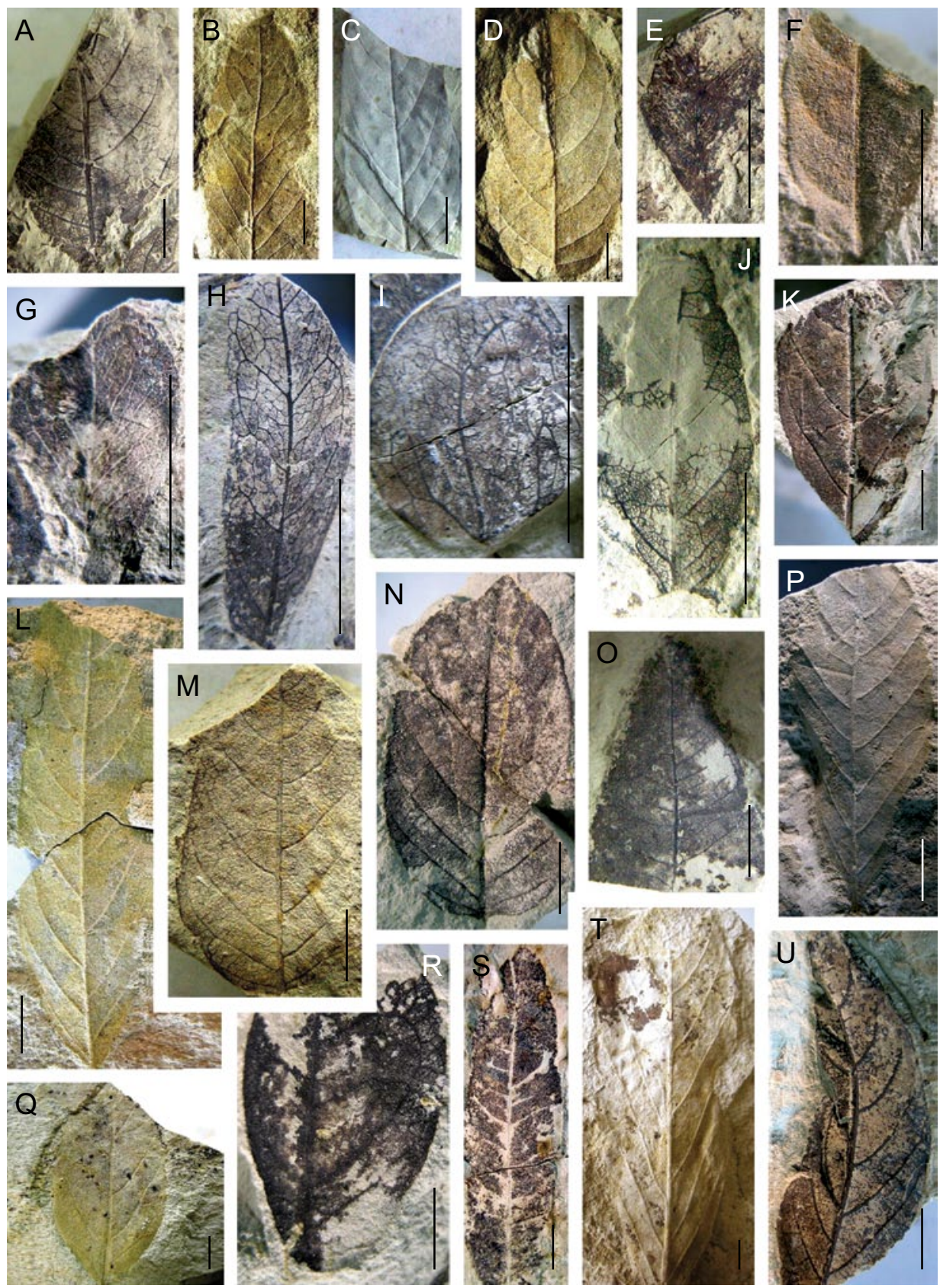

Fig. 8. Hojas del Mioceno de Tlaxcala, Chiapas. Material cortesía de Aldo Domínguez de la Torre. Escala $=1 \mathrm{~cm}$. 


\section{ACTIVIDAD VOLCÁNICA Y LA BIODIVERSIDAD}

Es importante comentar el amplio efecto que la actividad volcánica pudo tener sobre la vida y su evolución en México durante los últimos ca. 100 millones de años. El nuestro es un país de volcanes que se localizan sobre todo a lo largo del sector occidental de México, conformando cuatro provincias volcánicas, la Sierra Madre Occidental, la Sierra de la Giganta, la Faja Volcánica Transmexicana y la Sierra Madre del Sur (Ortega-Gutiérrez et al., 1992). En cada región esta actividad se ha desarrollado en distintos tiempos y con diferentes intensidades y modalidades, pero en conjunto se puede afirmar que estos eventos contribuyeron al desarrollo de los suelos, facilitaron u obstruyeron la ampliación de áreas de distribución de los organismos, afectaron la fisiografía de las regiones, modificaron las condiciones atmosféricas, incrementando el efecto de invernadero, etc. Aceptar que esta actividad incide en la evolución de las plantas es importante para entender la historia de las mismas; por ejemplo, una especie que en el Eoceno pudo tener una amplia distribución debió ser afectada por estos procesos de manera distinta dependiendo el tiempo y región en la que la interacción entre los organismos y su medio se dio. Los conceptos de tiempo y espacio son muy importantes para lograr una visión integral de los procesos que impulsan y generan biodiversidad. Así, la Sierra Madre Occidental en una primera etapa se desarrolló bajo un vulcanismo del tipo efusivo hasta hace ca. 32 millones de años, cuando este cambió a explosivo y contribuyó a extenderse e incrementar su altura, generó la mayor cubierta de ignimbritas preservada en el planeta (Bryan, 2007). La construcción de esta cadena montañosa da una idea de la enorme actividad volcánica de ese tiempo, así como del impacto que tuvo en la fisiografía y atmósfera. Su intensidad modificó el espacio que los organismos ocupaban. Esta Sierra no apareció repentinamente, se construyó de norte a sur a lo largo de ca. 60 millones de años, haciendo que su orogenesis en tiempo y espacio influyera sobre las poblaciones que habitaban en su entorno.

Otro ejemplo importante para entender la influencia de los volcanes en la vegetación lo ofrecen los estudios recientes de varios aparatos volcánicos en la Faja Volcánica Transmexicana. Su estudio y comparación hace evidente que como barrera o sendero biogeográfico esta Faja es relativamente reciente ( $\sim 20$ millones de años al presente), y que se formó por segmentos. Cada porción está compuesta por varios centros volcánicos que se originaron en momentos distintos y solo después de varios millones de años la provincia volcánica realmente representó una estructura que podía servir de barrera, pues antes de ello es probable que presentara varias "zonas de conexión" que comunicaban a veces a sitios distintos. Se ha calculado que en la 
Faja Volcánica Transmexicana existen ca. 8000 centros volcánicos (Gómez Tuena et al., 2005) formados en los últimos $\sim 20$ millones de años, evidenciando otra vez una tremenda actividad modificadora del entorno físico. Posiblemente buena parte de los ca. $2200 \mathrm{~m}$ sobre el nivel del mar a los que se encuentran los Valles de México, Puebla, Tlaxcala y Toluca está muy relacionada a esta actividad volcánica; pero habrá que esperar más datos que confirmen este cálculo hasta hoy solo basado en la presencia de vegetación similar a una selva tropical alta en Tlaxcala, hace ca. 12 millones de años.

Toda esta actividad volcánica que evidentemente es fuente impulsora de procesos biológicos y generadora de biodiversidad, obligó a los organismos a adaptarse a cambios hidrológicos, edáficos, de temperatura y humedad, de calidad de luz, etc., que deben ser contrastados con las modificaciones que se dieron a lo largo de la planicie del Golfo de México, en donde la influencia del vulcanismo fue mínima, pero en donde la formación de cuencas de depósito originadas por los procesos tectónicos durante la construcción de la Sierra Madre Oriental y el retiro de los mares, igualmente generaron cambios que promovieron la formación gradual de nuevas especies, a través de procesos intermitentes de regresiones marinas.

Finalmente, para generar una mejor idea de los procesos que contribuyeron a incrementar la biodiversidad en México hay que considerar la formación de las penínsulas de Baja California y Yucatán, y el cierre del Istmo de Panamá. Esto ocurrió hace relativamente poco tiempo. El proceso de rompimiento de la Península de Baja California inició hace menos de 12 millones de años y la formación del Mar de Cortés hace menos de 6 millones de años (Atwater, 1970). La Península de Yucatán empezó a emerger hace ca. 5 millones de años. Poco después, aparentemente hace $\sim 3.5$ millones de años, el Istmo de Panamá ya pudo funcionar como puente entre las dos Américas, Norte y Sur. Estos procesos geológicos cambiaron la intensidad y posiblemente dirección de corrientes marinas que llevan humedad al continente y por lo mismo afectaron a las comunidades vegetales. Aún hay mucho que entender sobre la influencia de tales desenvolvimientos que impulsan la diversificación de la vegetación, pero son aspectos que debemos considerar en nuestras discusiones sobre la aparición y extinción de especies a través del tiempo.

Recientemente se ha puntualizado el desarrollo de biomas en el Mioceno, que fueron cambiando de área geográfica conforme las condiciones del medio se modificaron, así se explica la aparición de las selvas secas a partir de selvas tropicales (Schrire et al., 2005a,b; Pennington et al., 2009). En esta propuesta, Mesoamérica es importante y sirve como puente para el establecimiento o aumento de distribución de los biomas o grandes floras. Sin embrago, y solo como ejemplos que invitan 
a continuar elaborando esta idea hay que comentar algunos supuestos aún débiles en la propuesta. Mesoamérica no existía como tal hace ca. 24 ma y el puente entre Norte y Sur América se estableció hace ca. $3.8 \mathrm{ma}$, entonces es probable que las selvas tropicales que dan lugar a biomas secos en América del Norte estuvieron en esta región desde antes, en el Paleógeno (65-32 ma), y los cambios fisiográficos del Neógeno favorecieron su desarrollo. Esto lo sugiere el registro fósil de México, por ejemplo Inga (Leguminosae) se conoce de rocas con edad de ca. 50 millones de años en La Popa, Nuevo León, y su aumento en diversidad y área de distribución se hace evidente en el Oligoceno de Tepexi de Rodríguez, Puebla, donde otra especie del mismo género está presente (Magallón Puebla y Cevallos-Ferriz, 1994b; CalvilloCanadell y Cevallos-Ferriz, 2002, 2005). Algo semejante sugieren la presencia de Tapirira, Haplorhus y Loxopterigium (Anacardiaceae) que documentan un proceso de ampliación de sus áreas de distribución hacia el sur a partir de Norte América desde hace ca. 50 millones de años (Ramírez y Cevallos-Ferriz, 2000a,b;), y ellas hoy son ejemplos de la llamada Flora Neotropical, que como los fósiles sugieren tenía representantes en América del Norte desde mucho antes de que el puente entre las dos Américas se formara, y entonces existiera un continuo continental capaz de contener a un tipo de flora que ahora reconocemos como la Flora Neotropical. La presencia de Lonchocarpus y Prioria (Leguminosae), otros miembros de la flora Neotropical, en sedimentos del Mioceno-Oligoceno de Tlaxcala y Puebla, con edad de ca. 12 y 30 ma respectivamente, es otro ejemplo que requiere de la existencia de Mesoamérica para lograr una distribución continua en América, y su presencia en América del Norte desde el Paleógeno reafirma la importancia que pudo tener México para la generación de taxa que inician su desarrollo en el trópico húmedo y se expanden hacia el trópico seco en el pasado (Hernández-Damián, 2010; SainzReséndiz, 2011).

Posiblemente otros dos ejemplos de plantas comunes hoy en día en México pero con escaso registro fósil ayuden a comprender la relación entre las formas de vida, su asociación y la evolución geológica. Pinus incluye a una serie de plantas que forman varios tipos de comunidades, y en diferentes zonas geográficas distintas especies son dominantes. Su diversidad y amplia distribución geográfica en México suponen una larga historia. Sin embrago, si bien las coníferas en México se han recolectado en sedimentos del Mesozoico, especialmente del Cretácico, y se han descrito miembros de Pinaceae, o cercanamente relacionados a esta familia, Pinus solo se reconoce con base en granos de polen desde el Eoceno, y sus macrofósiles aún no se han recolectado. Se ha propuesto que desde el Eoceno Pinus ocupaba las zonas altas de México (Martínez-Hernández y Ramírez-Arriaga, 1996), otros nos 
inclinamos a pensar que si bien si había zonas altas en México, su presencia debe documentarse con macro fósiles, que son relativamente propensos a fosilización. Posiblemente el polen proviene de otras zonas geográficas, o se distribuía en zonas altas pero de forma muy restringida. Esta historia tiene aún mucho que ofrecer para explicar cómo es que Pinus es en la actualidad tan diverso en el país. Si se tiene que generar una idea con los datos actuales, se podría adelantar que es la diversificación de los escenarios debido a la actividad geológica durante el Neógeno (MiocenoReciente) la que impulsa la diversificación y biodiversidad actual.

En el sur de México las evidencias sugieren que coníferas hoy más comunes en Asia, Cupressaceae y Pinaceae, crecieron desde el Cretácico y continuaron durante el Paleógeno, no encontrándose evidencia de macrofósiles de Pinus sino hasta hace relativamente poco tiempo, sugiriendo que la diversificación de este último taxon es reciente y probablemente ligado a la evolución geológica de las sierras en donde es común.

De manera similar, los encinos tan abundantes en ciertas regiones de México, no aparecen en el registro fósil sino hasta el Oligoceno, en donde su polen se hace más común. De forma semejante a la historia de los pinos, los macro fósiles de encinos, hojas y madera, son comunes solo a partir del Plioceno. La historia de estos dos taxa ejemplifica la relación entre la geología y la biología, además de resaltar la importancia de estudiar y ver a la naturaleza como un sistema, el Sistema Tierra.

\section{CONCLUSIONES}

Existen muchos espacios por completar con relación a la historia de la vegetación de México. Los estudios del Cuaternario continúan mostrando cambios en las comunidades del pasado, dejan claro la rapidez con que estas modificaciones pueden llevarse a cabo, y resaltan cómo el cambio climático recurrente asociado a las glaciaciones modeló la flora actual. A lo largo de la Faja Volcánica Mexicana se han realizado gran número de estudios en las últimas décadas y los cambios de bosques húmedos a pastizales o bosques mesófilos dominados por pinos, encinos o mezclas de ambos es muy común, y estas transformaciones pueden claramente ligarse con cambios en la fisiografía y clima. Desde luego que las escalas de comparación de tales estudios con aquellos pre Cuaternarios difieren mucho, pero están de acuerdo en que los procesos geológicos son modeladores de la fisiografía y entonces modificadores del clima, convirtiéndose en muy importantes para detonar procesos biológicos que dieron como resultado la gran biodiversidad que hoy hace a México especial en 
cuanto a ser un catálogo envidiable de formas de vida, que tiene mucho que aportar aún para entender de manera global la riqueza biológica de la que somos parte (e.g., Caballero et al., 2010; Israde Alcántara et al., 2010; Sosa-Najera et al., 2010).

Reconocer las aportaciones de los estudios filogeográficos a la visión histórica del origen de la biodiversidad es importante, ya que permiten conocer el avance de los desarrollos de especiación de un taxon, mismos que pueden ser discutidos junto con algún(os) proceso(s) geológico(s) (Ortiz-Medrano et al., 2008; RamírezBarahona, 2009; Sotuyo et al., 2010). Sin embargo, es necesario contar con información sobre la evolución geológica de las regiones en cuestión, y desafortunadamente estos estudios detallados están aún por realizarse en México, aunque sí existen fuentes importantes de datos que incidirán en la generación de una visión interdisciplinaria de la biodiversidad. Finalmente, estas hipótesis deben buscar sustento en el registro fósil que es la única fuente de información directa de la vida pasada.

\section{AGRADECIMIENTOS}

Los autores reconocen pláticas y discusiones con colegas que enriquecieron las ideas presentadas; asimismo, agradecen a los estudiantes que a través de tesis, trabajo de campo y participación en el Taller Sistemática Vegetal en el Tiempo han aportado materiales e ideas sobre la historia de la biodiversidad en México. El trabajo fue apoyado económicamente por los proyectos del Consejo Nacional de Ciencia y Tecnología, 104515 a LCC y 82433 a SRSCF, y el PAPIIT-UNAM 219810 a SRSCF, instituciones a las que se les agradece su apoyo. La ayuda técnica de Enoch Ortiz Montejo ha sido fundamental para el estudio de los fósiles que aquí se mencionan.

\section{LITERATURA CITADA}

Atwater, T. 1970. Implications of plate tectonics for the Cenozoic tectonic evolution of Western North America. Geol. Soc. Amer. Bull. 81(12): 3513-3536.

Bateman, R. M. y W. A. DiMichele. 2003. Genesis of phenotypic and genotypic diversity in land plants: The present as the key to the past. Syst. Biodiv. 1: 13-28.

Bonfante, P. y M-A. Selosse 2010. A glimpse into the past of land plants and of their mycorrhizal affairs: From fossils to evo-devo. New Phytol. 186: 267-270.

Bradley, R. S., K. Alverson y T. F. Pederson. 2003. Challenges of a changing earth: Past perspectives, future concerns. In: Alverson, K., R. S. Bradley y T. F. Pederson (eds.). Paleoclimate, global change and the future. Springer Verlag. Berlin, Alemania. pp. 163-167. 
Bryan, S. 2007. Silicic large igneous provinces. Episodes 30(1): 20-31.

Burns, B. P., R. Anitori, P. Butterworth, R. Henneberger, F. Goh, M. A. Allen, R. IbañezPeral, P. L. Bergquist, M. R. Walter y B. A. Neilan. 2009. Modern analogues and the early history of microbial life. Precambrian Res. 173: 10-18.

Caballero, M., S. Lozano-García, L. Váquez-Selem y B. Ortega. 2010. Evidencias de cambio climático y ambiental en registros glaciales y en cuencas lacustres del centro de México durante el último máximo glacial. Bol. Soc. Geol. Mex. 62(3): 359-377.

Calvillo-Canadell, L. y S. R. S. Cevallos-Ferriz. 2002. Bauhcis moranii gen. et sp. nov. (Cercideae, Caesalpinieae) an Oligocene plant from Tepexi de Rodríguez, Puebla, Mex., with leaf architecture similar to Bauhinia and Cercis. Rev. Palaeob. Palynol. 122(3-4): 171-184.

Calvillo-Canadell, L. y S. R. S. Cevallos-Ferriz. 2005. Diverse assemblage of Eocene and Oligocene Leguminosae from Mexico. Int. J. Plant Sci. 166: 671-692.

Calvillo-Canadell, L. y S. R. S. Cevallos-Ferriz. 2007. Flowers of Rhamnaceae from the Cerro del Pueblo (Upper Cretaceous, Coahuila) and Coatzingo (Oligocene, Puebla) formations, Mexico. Am. J. Bot. 94(10): 1658-1669.

Calvillo-Canadell, L. y S. R. S. Cevallos-Ferriz y L. Rico-Arce. 2010. Legume flowers preserved in amber form Simojovel de Allende Chiapas, Mexico. Rev. Palaeob. Palynol. 160: 126-134.

Calvillo-Canadell, L., O. J. Rodríguez-Reyes, R. Medina-Lemos y S. R. S. Cevallos-Ferriz. (sometido). Leaflets of Bursera (Burseraceae) in Eocene sediments from La Popa, Nuevo León, La Carroza Formation, Mexico. Am. J. Bot.

Castañeda-Posadas, C. y S. R. S. Cevallos-Ferriz. 2007. Swietenia (Meliaceae) flower included in Miocene amber from Simojovel de Allende, Chiapas, Mexico. Am. J. Bot. 94(11): 1821-1827.

Cevallos-Ferriz, S. R. S. 1983. Descripción de una madera de angiosperma cretácica de Cananea, Son., Méx. Los xilitos en el estudio del origen de las Angiospermopsida. An. Inst. Biol. UNAM 54: 97-112.

Cevallos-Ferriz, S. R. S. y L. Calvillo-Canadell. 2010. Ámbar, recinto de vida, resguardo de biodiversidad. Dirección General de Publicaciones y Fomento Editorial, Universidad Nacional Autónoma de México. México, D.F., México. 207 pp.

Cevallos-Ferriz, S. R. S. y E. A. González-Torres. 2005. Geological setting and phytobiodiversity in Mexico. In: Vega, F. J., T. G. Nyborg, M. C. Perrilliat, M. Montellano, S. Cevallos y S. Quiroz (eds.). Studies on Mexican paleontology. Springer. Dordrecht, Holanda. pp. 1-15.

Cevallos-Ferriz, S. R. S. y R. Weber. 1992. Dicotyledonous wood from the Upper Cretaceous (Maestrichtian) of Coahuila. Rev. Inst. Geol. 10: 65-70.

Collinson, M. E. 2002. The ecology of Cainozoic ferns. Rev. Palaeobot. Palynol. 119: 51-68.

Crane, P. R., P. Herendeen y E. M. Friis. 2004. Fossils and plant phylogeny. Am. J. Bot. 91: 1683-1699.

Domínguez de la Torre, A., L. Calvillo-Canadell, V. López Gómez y S. R. S. CevallosFerriz. 2011. Clima en San Esteban Tizatlán, Tlaxcala, durante el Mioceno con base en un análisis de arquitectura foliar. Resúmenes del XII Congreso Nacional de Paleontología. Puebla, México. 
Dornbos, S. Q. y S. Xiao. 2008. The dawn of animal life: Evolutionary and palaeoecological patterns in the Neoproterozoic-Cambrian animal fossil record. Palaeogogr. Palaoecl. Palaeoecol. 258: 135-137.

Eguiluz de Antuñano, S., M. Aranda-García y R. Marrett. 2000. Tectónica de la Sierra Madre Oriental, México. Bol. Soc. Geol. Mex. 53: 1-26.

Estrada-Ruiz, E., H. I. Martínez-Cabrera y S. R. S. Cevallos-Ferriz. 2010. Upper Cretaceous woods from the Olmos Formation (Late Campanian-Early Maastrichtian), Coahuila, Mexico. Am. J. Bot. 97(7): 1179-1194.

Estrada-Ruiz, E., H. I. Martínez-Cabrera y S. R. S. Cevallos-Ferriz. 2007. Fossil woods from the late Campanian-early Maastrichtian Olmos Formation, Coahuila, Mexico. Rev. Palaeobot. Palynol. 145: 123-133.

Estrada-Ruiz, E., G. R. Jr., Upchurch y S. R. S. Cevallos-Ferriz. 2008. Flora and climate of the Olmos Formation (Upper Campanian-Lower Maastrichtian), Coahuila, Mexico: a preliminary report. Trans. GCAGS 58: 273-283.

Estrada-Ruiz, E., G. R. Jr., Upchurch, J. A. Wolfe y S. R. S. Cevallos-Ferriz. 2011. Comparative morphology of fossil and extant leaves of Nelumbonaceae, including a new genus from the Late Cretaceous of western North America. Syst. Bot. 36(2): 337-351.

Feild, T. S. y N. C. Arens 2007. The ecophysiology of early angiosperms. Plant Cell Environ. 30: 291-309.

Ferrari, L., M. López-Martínez, G. Aguirre-Díaz y G. Carrasco-Nuñez 1999. Spacetime patterns of Cenozoic arc volcanism in central Mexico: from the Sierra Madre Occidental to the Mexican Volcanic Belt. Geology 27: 303-306.

Ferrari, L., M. Valencia-Moreno y S. Bryan. 2005. Magmatismo y tectónica de la Sierra Madre Occidental y su relación con la evolución de la margen occidental de Norteamérica. In: Nieto-Samaniego, A. y S. Alaniz-Álvarez (eds.). Volumen Conmemorativo del Centenario, Temas Selectos de la Geología Mexicana. Bol. Soc. Geol. Mex. 57(3): 343-378.

Frohlich, M. W. y M. W. Chase. 2007. After a dozen years of progress the origin of angiosperms is still a great mystery. Nature 450: 1184-1189.

Goldhammer, R. 1999. Mesozoic sequence stratigraphy and paleogeographic evolution of northeast Mexico. In: Bartolini, C., J. Wilson y T. Lawton (eds.). Mesozoic Sedimentary and Tectonic History of North-Central Mexico. Geol. Soc. Amer., Spec. Paper 340: 1-58.

Gómez Tuena, A., T. Orozco-Esquivel y L. Ferrari. 2005. Petrogénesis ígnea de la Faja Volcánica Transmexicana. Bol. Soc. Geol. Mex. 57(3): 227-285.

González-Ramírez, I., L. Calvillo-Canadell, A. Silva- Pineda y S. R. S. Cevallos-Ferriz. 2011. Coníferas (Araucariaceae y Cupressaceae) del Cretácico (Aptiano-Campaniano) de Chiapas. Resúmenes del XII Congreso Nacional de Paleontología. 22-25 feb, 20011, Puebla, México.

Guerrero-Márquez, G., L. Calvillo-Canadell y S. R. S. Cevallos-Ferriz. 2011. Angiospermas de la localidad El Chango, Aptiano-Campaniano, de Chiapas, México. Resúmenes del XII Congreso Nacional de Paleontología. 22-25 feb, 20011, Puebla, México.

Hernández-Castillo, G. R. y S. R. S. Cevallos-Ferriz. 1999. Reproductive and vegetative organs with Haloragaceae affinity from the Huepac chert, Upper Cretaceous of Sonora, Mexico. Amer. J. Bot. 86: 1717-1734. 
Hernández-Damián, A. L. 2010. Diversidad foliar en el Mioceno de San Esteban Tizatlán, Tlaxcala, México. Tesis de licenciatura. Facultad de Ciencias, Universidad Nacional Autónoma de México. México, D.F. México. 68 pp.

Hilton J. y R. M. Bateman. 2006. Pteridosperms are the backbone of seed-plant phylogeny. J. Torrey Bot. Soc. 133: 119-168.

Huerta-Vergara A. R., L. Calvillo-Canadell, A. Silva-Pineda y S. R. S. Cevallos-Ferriz. 2011. Fascículo foliar Cretácico de Pinaceae de la localidad del Chango, Chiapas, México. Resúmenes XII Congreso Nacional de Paleontología. 22-25 feb, 20011, Puebla, México.

Israde Alcántara I., R. Velázquez-Durán, M. S. Lozano García, J. Bischoff, G. Domínguez Vázquez y V. H. Garduño Monroy. 2010. Evolución paleolimnológica del Lago Cuitzeo, Michoacán durante el Pleistoceno-Holoceno. Bol. Soc. Geol. Mex. 62(3): 345-357.

Jasso-Castañeda C., S. Sedov, E. Solleiro-Rebolledo y J. Gama-Castro. 2002. El desarrollo de paleosuelos como índice de estabilidad del paisaje. Invest. Geog. 47: 20-35.

Lewis, S. L. 2006. Tropical forests and the changing Earth system. Phil. Tran. Royal Soc. B: Biol. Sci. 361: 195-210.

Lieberman, B. S. 2000. Palaeobiogeography: Using fossils to study global change, plate tectonics, and evolution. Kluwer Academic/Plenum Publishers. Nueva York, USA. 208 pp.

Linkies A., K. Graeber, C. Knight y G. Leubner-Metzger 2010. The evolution of seeds. New Phytol. 186: 817-831.

Long A. G. 1977. Observations on Carboniferous seeds of Mitrospermum, Conostoma and Lagenostoma. Trans. R. Soc. Edin. 70: 37-61.

Magallón-Puebla S. y S. R. S. Cevallos-Ferriz. 1994a. Latest occurrence of the extinct genus Cedrelospermum (Ulmaceae) in America: Cedrelospermum manchesteri sp. nov. from the Upper Cenozoic of Puebla, México. Rev. Palaeobot. Palynol. 81: 115-128.

Magallón-Puebla, S. y S. R. S. Cevallos-Ferriz. 1994b. Fossil legume pods from Tertiary strata of Tepexi de Rodríguez, Puebla, Mexico. Can. J. Bot. 72: 1027-1038.

Magallón-Puebla S. y S. R. S. Cevallos-Ferriz. 994c. Eucommia constans n. sp. fruits from the upper Cenozoic strata of Puebla, Mexico: morphological and anatomical comparison with Eucommia ulmoides Oliver. Int. J. Plant Sci. 155: 80-95.

Maldonado-Koerdell, M. 1950. Los estudios paleobotánicos en México, con un catálogo sistemático de sus plantas fósiles. Bol. Inst. Geol. 55: 1-72.

Martínez-Cabrera, H. I. y S. R. S. Cevallos-Ferriz. 2006. Maclura (Moraceae) wood from the Miocene of the Baja California Peninsula, Mexico: Fossil and biogeographic history of its closer allies. Rev. Palaeobot. Palynol. 140: 113-122.

Martínez-Cabrera, H. I., S. R. S. Cevallos-Ferriz y I. Poole. 2006. Fossil woods from Early Miocene sediments of the El Cien Formation, Baja California Sur, Mexico. Rev. Palaeobot. Palynol. 138: 141-163.

Martínez-Hernández, E. y E. Ramírez-Arriaga. 1996. Paleocorología de angiospermas de la flora mexicana durante el Mesozoico y Terciario: Algunas evidencias palinológicas. Bol. Soc. Mex. Bot. 58: 87-97.

Maruyama, S. y M. Santosh. 2008. Snowball Earth to Cambrian explosion. Gondwana Res. 14: 1-4. 
Méndez-Cárdenas J., S. R. S. Cevallos-Ferriz y L. Calvillo-Canadell. (sometido). Variabilidad y el concepto de género forma ejemplificado por Paraphyllanthoxylon. Rev. Mex. Cienc. Geol.

Miranda, F. 1963. Two plants from the amber of Simojovel, Chiapas, Mexico, area. J. Paleontol. 37: 611-614.

Morán-Zenteno, D. 1984. Geología de la República Mexicana. Universidad Nacional Autónoma de México, Instituto Nacional de Estadística, Geografía e Informática (INEGI). México, D.F., México. 88 pp.

Nixon, K. C. 1996. Paleobotany in cladistics and cladistics in paleobotany: Enlightenment and uncertainty. Rev. Palaeobot. Palynol. 90: 361-373.

Ortega-Gutiérrez, F., L. Mitre-Salazar, J. Roldan-Quintana, J. Aranda-Gómez, D. MoránZenteno, S. Alaniz-Álvarez y A. Nieto-Samaniego. 1992. Texto explicativo de la 5a. edición de la carta geológica de la República Mexicana Escala 1:2 000 000, Instituto de Geología, Universidad Nacional Autónoma de México, Consejo de Recursos Minerales, Secretaría de Energía, Minas e Industria Paraestatal. México, D.F., México. $74 \mathrm{pp}$.

Ortíz-Medrano, A., A. Moreno-Letelier y D. Piñero. 2008. Fragmentación y expansión demográfica en las poblaciones mexicanas de Pinus ayacahuite var. ayacahuite. Bol. Soc. Bot. Méx. 83: 25-36.

Padilla, J. y R. Sánchez. 2007. Evolución geológica del sureste mexicano desde el Mesozoico al presente en el contexto regional del Golfo de México. Bol. Soc. Geol. Mex. 69(1): 19-42.

Palacios Chávez, R. y J. Rzedowski. 1993. Estudio palinológico de las floras fósiles del Mioceno Inferior y principios del Mioceno Medio de la región de Pichucalco, Chiapas, México. Acta Bot. Mex. 24: 1-96.

Pennington, R. T., M. Lavin y A. Oliveira-Filho. 2009. Evolution, and ecology in the tropics: perspectives from seasonally dry tropical forests. Annu. Rev. Ecol. Evol. Syst. 40: 437-457.

Pennisi, E. 2009. On the origin of flowering plants. Science 324: 28-3.

Peralta-Medina, E. 2009. Arquitectura foliar de hojas fósiles de Ixtapa, Chiapas, México. Tesis de licenciatura. Facultad de Ciencias, Universidad Nacional Autónoma de México. México, D.F., México. 67 pp.

Phillips, T. L. y W. A. DiMichele. 1992. Comparative ecology and life-history biology of arborescent lycopsids in Late Carboniferous swamps of Euramerica. Ann. Mo. Bot. Gard. 79: 560-588.

Ramírez, J. L. y S. R. S. Cevallos-Ferriz. 2000a. Leaves of Salicaceae (Salix and Populus) from Oligocene sediments, near Tepexi de Rodríguez, Puebla. Int. J. Plant Sci. 161: 521-534.

Ramírez, J. L. y S. R. S. Cevallos-Ferriz. 2000b. Leaves of Berberidaceae (Berberis and Mahonia) from Oligocene sediments, near Tepexi de Rodríguez, Puebla. Rev. Paleobot. Palynol. 110: 247-257.

Ramírez, J. L. y S. R. S. Cevallos-Ferriz. 2002. A diverse assemblage of Anacardiaceae from Oligocene sediments, Tepexi de Rodríguez, Puebla, Mexico. Am. J. Bot. 89: 535-545.

Ramírez, J. L., S. R. S. Cevallos-Ferriz y A. Silva Pineda. 2000. Reconstruction of the leaves of two new species of Pseudosmodingium from Oligocene strata of Puebla, Mexico. Int. J. Plant Sci. 161: 509-519. 
Ramírez-Barahona, S. A. 2009. Variación genética y filogeografía de helechos arborescentes (Cyatheaceae) del bosque mesófilo de montaña de la Sierra Madre Oriental. Tesis de licenciatura. Facultad de Ciencias, Universidad Nacional Autónoma de México. México, D.F., México. 128 pp.

Rodríguez de la Rosa, R. A. y S. R. S. Cevallos-Ferriz. 1994. Upper Cretaceous zingiberalean fruits with in situ seeds from southeast Coahuila, Mexico. Int. J. Plant Sci. 155: 786805.

Rodríguez de la Rosa, R. A., S. R. S. Cevallos-Ferriz y A. Silva-Pineda. 1998. Paleobiological implications of Campanian coprolites. Palaeogogr. Palaoecl. Palaeoecol. 142: 231254.

Rodríguez-Reyes, O. J. 2007. Material foliar del Eoceno de la Fm. Carroza en La Popa, Nuevo León, México. Tesis de maestría. Posgrado en Ciencias Biológicas, Universidad Nacional Autónoma de México. México, D.F., México. 79 pp.

Royer, D. L. 2010. Fossil soils constrain ancient climate sensitivity. Proc. Natl. Acad. Sci. 107: 517-518.

Russell, M. 2006. First life. Am. Sci. 94: 32-39.

Sainz-Reséndiz, B. A. 2011. Descripción e identificación de maderas del Paleógeno de San Juan Atzingo, Puebla, México. Tesis de licenciatura. Facultad de Estudios Superiores, Iztacala. Universidad Nacional Autónoma de México. México, D.F., México. 63 pp.

Sanders, H., G. W. Rothwell y S. E. Wyatt 2009. Key morphological alterations in the evolution of leaves. Int. J. Plant Sci. 170: 860-868.

Schopf, J. W. 2006. The first billion years: when did life emerge? Elements 2: 229-233.

Schrire, B. D., M. Lavin y G. P. Lewis. 2005a. Biogeography of the Leguminosae. In: Lewis, G., B. Schrire, B. Mackinder y M. Lock (eds.). Legumes of the World. Royal Botanic Gardens. Kew, UK. pp. 21-54.

Schrire, B. D., M. Lavin y G. P. Lewis. 2005b. Global distribution patterns of the Leguminosae: insights from recent phylogenies. In: Friis, I. y H. Balslev (eds.). Plant diversity and complexity patterns. Danske Videnskabernes Selskab, Biologiske Skrifter 55: 375-422.

Silva-Pineda, A. y B. E. Buitrón-Sánchez. 1999. Mesozoic redbed floras in east-central Mexico and their stratigraphic relationships with marine beds. Geol. Soc. Am Spec. Paper 340: 151-160.

Smith, S. A., J. M. Beaulieu y M. J. Donoghue. 2010. An uncorrelated relaxed-clock analysis suggests an earlier origin for flowering plants. Proc. Natl. Acad. Sci. 107: 5897-5902.

Soeegard, K. H., Y. E. Kalik, A. T. Daniels, J. Arney y S. Garrick. 2003. Stratigraphic evolution of the Latest Cretaceous to early Tertiary Difunta foreland basin in northeast Mexico: Influence of salt withdrawal on tectonically induced subsidence by the Sierra Madre Oriental fold and thrust belt. In: Bartolini, C., R. T. Buffler y J. Blickwede (eds.). The Circum-Gulf of Mexico and the Caribbean Hydrocarbon habitats, basin formation and plate tectonics. AAPG Memoir 79: 364-394.

Sosa-Najera, S., S. Lozano-García, P. Roy y M. Caballero. 2010. Registro de sequías históricas en el occidente de México con base en el análisis elemental de sedimentos lacustres: El caso del Lago de Santa María del Oro. Bol. Soc. Geol. Mex. 62(3): 437451. 
Sotuyo, S., A. Delgado-Salinas, G. P. Lewis, M. W. Chase, L. Ferrari y K. Oyama. 2010. Filogeografía del complejo Caesalpinia hintonii: (Leguminosae: Caesalpinioideae: Poincianella). Rev. Mex. Biodiv. 81: 883-894.

Stubblefield, S. P. y G. W. Rothwell. 1980. Conostoma chappellicum n. sp., lagenostomalean ovules from Kentucky. J. Paleontol. 54: 1012-1016.

Stubblefield, S. P., G. W. Rothwell y T. N. Taylor. 1984. Conostoma williamsonii n. sp., a lagenostomalean ovule from the Pennsylvanian of the Illinois Basin. Can. J. Bot. 62: 96-101.

Taylor, T. N., E. L. Taylor y M. Krings. 2009. Paleobotany: The biology and evolution of fossil plants. 2a. ed. Academic Press. Burlington, USA. 1230 pp..

Urrutia-Fucugauchi, J. 2011. The Chicxulub multi-ring impact crater, Yucatan carbonate platform, Gulf of Mexico. Geofis. Inter. 50(1): 99-127.

van Konijnenburg-Van Cittert, J. H. A. 2002. Ecology of some Late Triassic to Early Cretaceous ferns in Eurasia. Rev. Palaeob. Palynol. 119: 113-124.

Vázquez-Rueda, M. A., L. Calvillo-Canadell, C. Y. Núñez-Mariel y S. R. S. Cevallos-Ferriz. 2011. Fruto del Cretácico Superior de la Formación Cerro del Pueblo, Coahuila. XII Congreso Nacional de Paleontología. Puebla, México.

Velasco de León, M. P. y S. R. S. Cevallos-Ferriz. 2000. Leaves of Cercocarpus mixteca $\mathrm{n}$. sp. (Rosaceae) from Oligocene sediments, near Tepexi de Rodríguez, Puebla. Rev. Palaeobot. Palynol. 111: 285-294.

Velasco-de León, M. P. y E. L. Ortiz-Martínez. 2010. Nuevas especies de Quercus (Fagaceae) en el Plioceno de Santa María Amajac, Hidalgo, México. Rev. Mex. Cien. Geol. 27(2): 264-277.

von Balthazar, M., J. Schönenberger y T. Denk. 2008. In search of the earliest flowers: Introduction. Int. J. Plant Sci. 169: 815.

Weber, R. 2008a. Plantas triásicas y jurásicas de México. Bol. Inst. Geol. 115: 1-84.

Weber, R. 2008b. Plantas triásicas y jurásicas de México. Bol. Inst. Geol. 115: 85-116.

Weber, R. 2008c. Plantas triásicas y jurásicas de México. Bol. Inst. Geol. 115: 117-149.

Weber, R. y S. R. S. Cevallos-Ferriz. 1995. Perfil actual y perspectivas de la paleobotánica en México. Bol. Soc. Bot. Méx. 55: 141-148.

Weng, J.-K. y C. Chapple. 2010. The origin and evolution of lignin biosynthesis. New Phytol. 187: 273-285.

Wilson, J. P. 2009. Green life through time. Science 325: 36-37. 